University of Nebraska - Lincoln

DigitalCommons@University of Nebraska - Lincoln

\title{
Remote sensing of phytoplankton community composition along the northeast coast of the United States
}

\author{
Xiaoju Pan \\ NASA Goddard Space Flight Center, xpanx001@gmail.com \\ Antonio Mannino \\ NASA Goddard Space Flight Center \\ Harold G. Marshall \\ Old Dominion University \\ Katherine C. Filippino \\ Old Dominion University \\ Margaret R. Mulholland \\ Old Dominion University
}

Follow this and additional works at: https://digitalcommons.unl.edu/nasapub

Part of the Physical Sciences and Mathematics Commons

Pan, Xiaoju; Mannino, Antonio; Marshall, Harold G.; Filippino, Katherine C.; and Mulholland, Margaret R., "Remote sensing of phytoplankton community composition along the northeast coast of the United States" (2011). NASA Publications. 79.

https://digitalcommons.unl.edu/nasapub/79

This Article is brought to you for free and open access by the National Aeronautics and Space Administration at DigitalCommons@University of Nebraska - Lincoln. It has been accepted for inclusion in NASA Publications by an authorized administrator of DigitalCommons@University of Nebraska - Lincoln. 


\title{
Remote sensing of phytoplankton community composition along the northeast coast of the United States
}

\author{
Xiaoju Pan a,*, Antonio Mannino a , Harold G. Marshall ${ }^{\text {b }}$, Katherine C. Filippino ${ }^{\text {c }}$, Margaret R. Mulholland ${ }^{\text {c }}$ \\ a NASA Goddard Space Flight Center, Greenbelt, MD 20771, USA \\ b Department of Biological Sciences, Old Dominion University, Norfolk, VA 23529, USA \\ c Department of Ocean, Earth, and Atmospheric Sciences, Old Dominion University, Norfolk, VA 23529, USA
}

\section{A R T I C L E I N F O}

\section{Article history:}

Received 25 January 2011

Received in revised form 20 June 2011

Accepted 9 September 2011

Available online 10 October 2011

\section{Keywords:}

CHEMTAX

Phytoplankton community

Remote sensing

United States northeast coast

MODIS-Aqua

\begin{abstract}
A B S T R A C T
Satellite imagery has proven to be a powerful tool for measuring chlorophyll $a$ in surface waters. While this provides an estimate of total phytoplankton biomass, it does not distinguish between phytoplankton groups, many of which have functional differences and therefore affect biogeochemical cycles differently. Phytoplankton pigment analysis has been used to quantify a wide range of photosynthetic and accessory pigments, and chemotaxonomic analysis (e.g. CHEMTAX) has been used to successfully quantify functional taxonomic groups in nature based on pigment distributions. Here, we combine CHEMTAX analysis with satellite-derived distributions of specific phytoplankton pigments to describe the distributions of particular components of the phytoplankton community in the northeast coast of the United States from space. The spatial and seasonal variations in phytoplankton community structure elucidated through satellite remote sensing methods generally agreed with observations of abundance estimates of cell counts. Diatoms were generally the most abundant phytoplankton in this region, especially during Winter-Spring and in the inner shelf, but phytoplankton populations shifted to increasing abundance of other taxa during Summer, especially offshore. While still preliminary, satellite-derived taxa-specific information with proper regional controls holds promise for providing information on phytoplankton abundance to a taxonomic group level which would greatly improve our understanding of the impacts of human activity and climate change on ecosystems.
\end{abstract}

(c) 2011 Elsevier Inc. All rights reserved.

\section{Introduction}

Satellite ocean color remote sensing is a valuable tool for studying large-scale variations of phytoplankton biomass which in turn affects biogeochemical cycling of elements (IOCCG, 1999, 2000; McClain, 2009). Global distributions of total chlorophyll a concentration ([TChl_a]) and extrapolations of primary production have been previously quantified from satellite observations, especially for the oceanic Case 1 waters (Behrenfeld \& Falkowski, 1997; Behrenfeld et al., 2005; McClain, 2009; O'Reilly et al., 1998, 2000). However, because of the limitations of satellite spectral resolution in distinguishing between plant pigments, the distribution and seasonal variation in phytoplankton community composition observed from satellite measurements are limited (Aiken et al., 2008, 2009; Alvain et al., 2008; Brewin et al., 2010, 2011; Hirata et al., 2008, 2011; McClain, 2009; Pan et al., 2010; Roy et al., 2011).

Phytoplankton community composition is important for determining biogeochemical function because different taxonomic groups have unique physiologies that affect their growth rates, $\mathrm{C}$ and $\mathrm{N}$

\footnotetext{
* Corresponding author at: Research Center for Environmental Changes, Academia Sinica, Taipei, Taiwan. Tel.: +886226539885x861; fax: +886227833584.

E-mail addresses: xpanx001@gmail.com,xpan@gate.sinica.edu.tw (X. Pan).
}

uptakes, competitive success, and biogeochemical functions in the environment. For example, blooms of large chain-forming diatoms can contribute disproportionately to the sinking biogenic particle flux of carbon than small phytoplankton such as cyanobacteria. Thus, a taxonomic level of analysis can improve our understanding of phytoplankton species distribution, primary productivity, and carbon export flux estimates (Aiken et al., 2008, 2009; Alvain et al., 2008; Brewin et al., 2011; Hirata et al., 2008, 2011; McClain, 2009; Roy et al., 2011; Uitz et al., 2009). This level of analysis is also useful in assessing regional variations in phytoplankton distribution as well as the effects of climate variability and change on the seasonal and spatial variation of phytoplankton species. For example, the distributions of phytoplankton functional groups, particularly dinoflagellates and prymnesiophytes, are often thought to correlate to dimethylsulfide (DMS), a precursor of cloud condensation nuclei (Townsend \& Keller, 1996).

Phytoplankton taxonomy is traditionally assessed by microscopy, which requires significant amounts of time and expertise. Species too fragile to be preserved or small in size (in particular, picophytoplankton) are challenging to identify by routine microscopic methods and this introduces biases to taxonomic assessments done using these methods. Advances in high-performance liquid chromatography (HPLC) methods have allowed the detection of an increasing number of phytoplankton pigments. This information can allow 
quantification of phytoplankton community composition, at least to a functional group level, because many plant pigments are particular to specific taxonomic groups or even species (Jeffrey et al., 1997; Mackey et al., 1996).

Chemotaxonomic assessment of phytoplankton assemblages has been further facilitated through development of algorithms to partition bulk [TChl_a] into taxonomic groups based on pigment composition of the community (Mackey et al., 1996). CHEMTAX (Mackey et al., 1996), a Matlab ${ }^{\circledR}$ (The Mathworks, Inc.) program, analyzes the relative pigment ratios for each taxonomic group, especially those pigments found only in one or two taxonomic groups (e.g. alloxanthin in cryptophytes, peridinin in peridinin-containing dinoflagellates, and zeaxanthin primarily in cyanobacteria and prochlorophytes; Jeffrey et al., 1997). However, CHEMTAX does not provide the same resolution as microscopy, because the latter can identify many groups to the species level. Overlapping pigment compositions can further complicate the capability for CHEMTAX to separate taxonomic groups, i.e. to separate chlorophytes from prasinophytes. Although there exist these limitations to CHEMTAX, it has been successful in describing variations of major phytoplankton taxonomic groups for many water types including: oceanic waters (e.g. the Southern Ocean and the northeastern Atlantic), and coastal waters (e.g. the New Jersey coast, the Chesapeake Bay estuary, United States southeastern estuaries, and European coastal waters) (Adolf et al., 2006; Gibb et al., 2001; Lewitus et al., 2005; Mackey et al., 1996; Moline \& Blackwell, 2004; Schlüter et al., 2000; Wright et al., 1996).

Studies of phytoplankton taxonomy are typically limited to discrete stations sampled during irregular ship surveys, and so we know little about the spatial and temporal variations of phytoplankton assemblages (Aiken et al., 2008, 2009; Alvain et al., 2008; Brewin et al., 2010; 2011; Hirata et al., 2008, 2011). Pan et al. (2010) showed that many important phytoplankton pigments could be empirically estimated from ocean color remote sensing within reasonable agreement to the field observations along the northeast coast of the United States (U.S.). In this study, phytoplankton community composition by chemotaxonomy (e.g. CHEMTAX) in the U.S. northeast coast was estimated using satellite retrieval of phytoplankton pigment composition (Pan et al., 2010).

The northeast coast of the U.S. is a highly productive and optically complex region that is significantly influenced by freshwater outflow (e.g. from the Chesapeake Bay, Delaware Bay, and Hudson-Raritan Rivers) and by alongshore flow and tidal forcing (Mannino et al., 2008; O'Reilly \& Zetlin, 1998; Pan et al., 2008, 2010). Phytoplankton species composition varies seasonally: diatoms generally dominate during Winter-Spring, while other taxa, such as dinoflagellates and cyanobacteria, increase in proportions during Summer (Adolf et al., 2006; Marshall, 1984a, 1984b; Marshall \& Cohn, 1987; Marshall et al., 2006; Moline \& Blackwell, 2004). The objectives of this study were: (1) to develop a quantitative method to describe phytoplankton community composition using satellite ocean color remote sensing; and (2) to determine the spatial and temporal variability of phytoplankton community composition, and its impact on biogeochemical processes in the U.S. northeast coast.

\section{Materials and methods}

\subsection{Field experiments}

This study focused on the U.S. northeast coast within the longitude and latitude boundaries of $77^{\circ} \mathrm{W}$ to $65^{\circ} \mathrm{W}$ and $35^{\circ} \mathrm{N}$ to $45^{\circ} \mathrm{N}$, respectively (Fig. 1). The study area includes the Gulf of Maine (GoM) and the Middle Atlantic Bight (MAB), in which the MAB is separated into the New York Bight (NYB) and the southern MAB (SMAB) (Fig. 1). Multiple cruises were conducted in this region from 2004 to 2009 (Table 1 and Fig. 1).

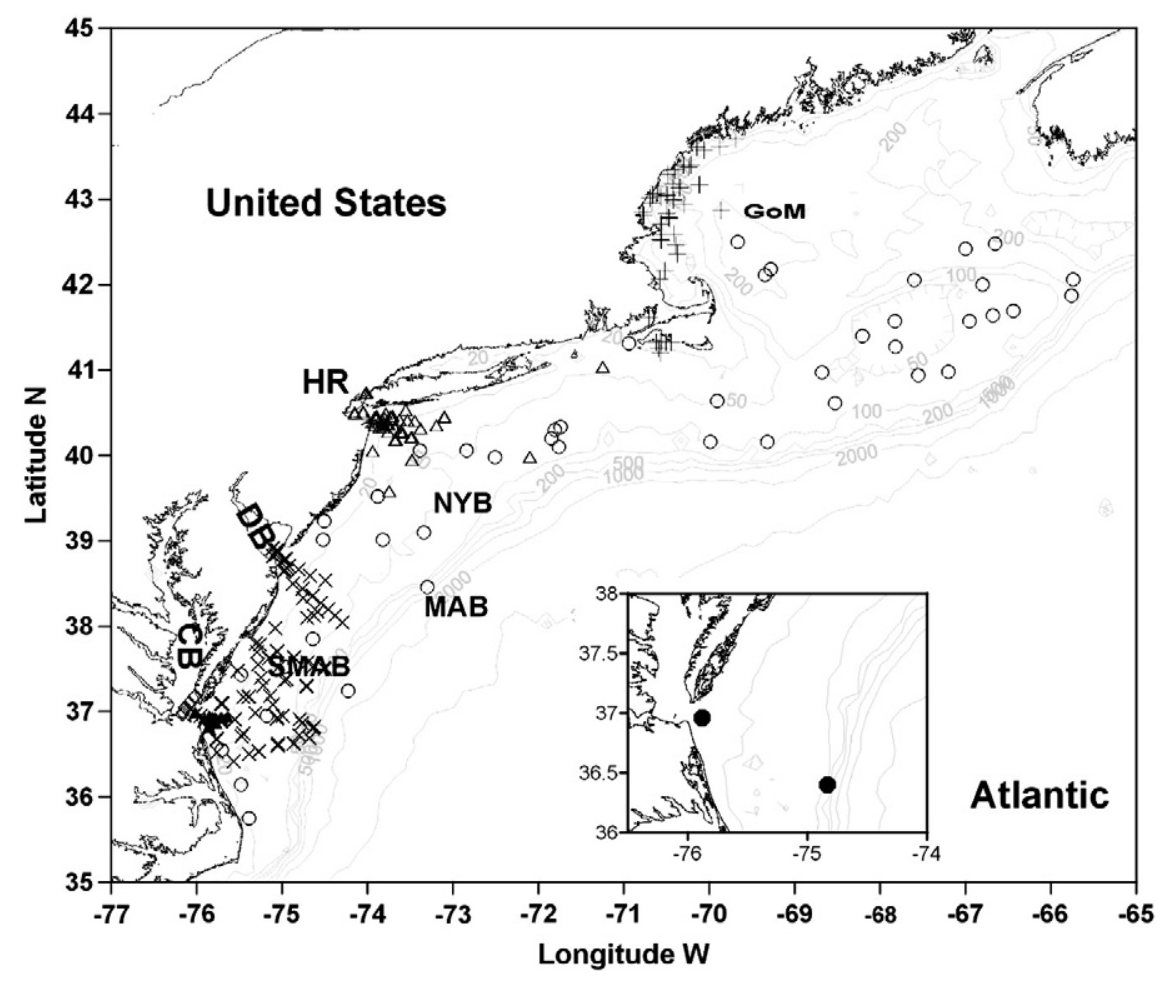

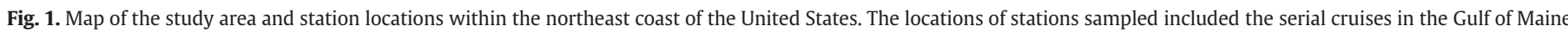

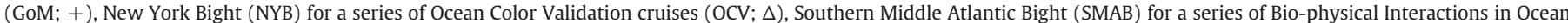

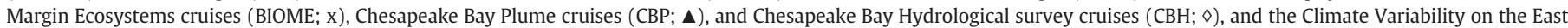

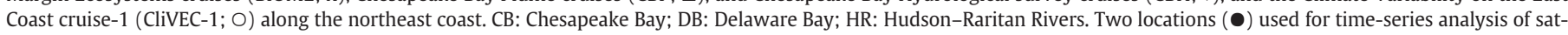
ellite derivations in the $\mathrm{CB}$ estuarine are shown in the inset. 
Table 1

Field cruises conducted for this study. GoM: Gulf of Maine; NYB: New York Bight; SMAB: southern Middle Atlantic Bight; OCV: Ocean Color Validation cruises; BIOME: Bio-physical Interactions in Ocean Margin Ecosystems cruises; CBP: Chesapeake Bay Plume cruises; CBH: Chesapeake Bay mouth Hydrological survey cruises; CliVEC-1: Climate Variability on the East Coast cruise-1.

\begin{tabular}{|c|c|c|c|c|}
\hline Regions & Cruises & Periods & Stations $^{\mathrm{a}}$ & Measurements $^{\mathrm{b}}$ \\
\hline \multirow[t]{5}{*}{ GoM } & GoM-1 & 26 to 30 Apr 2007 & $14 / 12 / 0$ & HPLC, $R_{r s}$ \\
\hline & GoM-2 & 26 to 28 May 2007 & $17 / 14 / 0$ & HPLC, $R_{\mathrm{rs}}$ \\
\hline & GoM-3 & 6 to 8 Jun 2007 & $13 / 12 / 0$ & HPLC, $\mathrm{R}_{\mathrm{rs}}$ \\
\hline & GoM-4 & 6 Sep 2007 & $5 / 5 / 0$ & HPLC, $\mathrm{R}_{\mathrm{rs}}$ \\
\hline & ClivEC $-1^{\mathrm{c}}$ & 17 to 28 Aug 2009 & $19 / 0 / 8$ & HPLC, CC \\
\hline \multirow[t]{5}{*}{ NYB } & OCV1 & 5 to 9 May 2007 & $20 / 19 / 0$ & HPLC, $\mathrm{R}_{\mathrm{rs}}$ \\
\hline & OCV2 & 10 to 14 Nov 2007 & $22 / 18 / 0$ & HPLC, $\mathrm{R}_{\mathrm{rs}}$ \\
\hline & OCV3 & 21 to $24 \mathrm{Jul} 2008$ & $8 / 8 / 0$ & HPLC, $R_{\mathrm{rs}}$ \\
\hline & OCV5 & 19 to 21 May 2009 & $10 / 0 / 0$ & HPLC \\
\hline & CliVEC $-1^{\mathrm{c}}$ & 17 to 28 Aug 2009 & $15 / 0 / 14$ & HPLC, CC \\
\hline \multirow[t]{8}{*}{ SMAB } & BIOME1 & 30 Mar to 1 Apr 2005 & $14 / 5 / 0$ & HPLC, $R_{r s}$ \\
\hline & BIOME2 & 26 to 30 Jul 2005 & $29 / 19 / 0$ & HPLC, $\mathrm{R}_{\mathrm{rs}}$ \\
\hline & BIOME3 & 9 to 12 May 2006 & $26 / 0 / 0$ & HPLC \\
\hline & BIOME4 & 2 to 6 Jul 2006 & $31 / 27 / 0$ & HPLC, $R_{\mathrm{rs}}$ \\
\hline & BIOME5 & 31 Oct 2006 & $5 / 0 / 3$ & HPLC, CC \\
\hline & $\mathrm{CBP}$ & $\begin{array}{l}27 \text { May, } 3 \text { Nov 2005; } \\
6 \text { Sep, } 28 \text { Nov 2006; } \\
3 \text { Jul } 2007\end{array}$ & $24 / 13 / 7$ & HPLC, $R_{\mathrm{rs}}, \mathrm{CC}$ \\
\hline & $\mathrm{CBH}$ & $\begin{array}{l}5 \text { May, } 5 \text { Jul, } 1 \text { Sep, } 15 \text { Oct, } \\
15 \text { Nov 2004; } 10 \text { Jan, } 26 \text { May, } \\
21 \text { Jun, } 19 \text { Aug, } 23 \text { Sep } 2005\end{array}$ & $35 / 0 / 0$ & HPLC \\
\hline & ClivEC $-1^{\mathrm{c}}$ & 17 to 28 Aug 2009 & $11 / 0 / 10$ & HPLC, CC \\
\hline
\end{tabular}

a Numbers of stations are shown on $\mathrm{N}_{1} / \mathrm{N}_{2} / \mathrm{N}_{3}$, while $\mathrm{N}_{1}, \mathrm{~N}_{2}$, and $\mathrm{N}_{3}$ represent how many stations during which HPLC, $\mathrm{R}_{\mathrm{rs}}$, and cell counting measurements were made, respectively.

b Selected parameters from the cruises, including HPLC phytoplankton pigments (HPLC), cell counting by microscopy or flow cytometry (CC), and remote sensing reflectance $\left(\mathrm{R}_{\mathrm{rs}}\right)$.

c CliVEC-1 was conducted along the northeast coast of the United States across the GoM, the NYB, and the SMAB.

\subsection{Pigment measurements and cell counts}

Pigment samples were filtered onto $25 \mathrm{~mm}$ GF/F filters, immediately frozen in liquid nitrogen, and stored frozen $\left(-80^{\circ} \mathrm{C}\right)$ until analysis (Pan et al., 2008, 2010). Samples were analyzed at Horn Point Laboratory (University of Maryland Center for Environment Science) using reverse-phase high-performance liquid chromatography (HPLC) with a C8 column and a photodiode array detector (Van Heukelem \& Thomas, 2001). The selected HPLC pigments analyzed include: total chlorophyll $a\left(T C h l \_a\right)$, divinyl Chl_a (DV_Chl_a), total chlorophyll $b$ (TChl_b), chlorophyll $c_{1}$ and $c_{2}\left(\mathrm{Chl} \_c_{1} / c_{2}\right)$, chlorophyll $c_{3}\left(\mathrm{Chl} \_c_{3}\right)$, fucoxanthin (Fuco), 19'-butanoyloxyfucoxanthin (But), 19'-hexanoyloxyfucoxanthin (Hex), peridinin (Perid), zeaxanthin (Zea), alloxanthin (Allo), diadinoxanthin (Diad) plus diatoxanthin (Diato) (Dia), lutein (Lut), neoxanthin (Neo), violaxanthin (Viola), and prasinoxanthin (Pras). TChl_a is defined as the sum of chlorophyll $a\left(C h l \_a\right), D V \_C h l \_a$, and chlorophyllide $a$ (Chlide_ $a$ ), while TChl $b$ is defined as the sum of chlorophyll $b$ (Chl $\_b$ ) and divinyl Chl_b (DV_Chl_b) (Hooker et al., 2005). Carotene (Caro) is not included in this analysis due to its rapid alteration to oxidized products. The abbreviations of pigments are also shown in Table 2.

Cell counts were conducted at Old Dominion University or University of Rhode Island using the method described by Marshall and Alden (1990). Water samples (500 mL or $1000 \mathrm{~mL}$ ) were fixed in Lugol's solution, and after a 48 hour period, a series (3) of siphoning and settling steps followed to obtain a $20-40 \mathrm{ml}$ concentrate that was placed in the settling chamber of an inverted plankton microscope (Nikon TS100) for analysis. The net-phytoplankton $(>200 \mu \mathrm{m})$ were scanned at $150 \times$ for the entire aliquot. A minimum cell count basis of 200 cells and 10 random fields was used at scanning at $300 \times$ for both the micro- $(20-200 \mu \mathrm{m})$ and nano-phytoplankton (2$20 \mu \mathrm{m})$. This analysis produced an $85 \%$ accuracy estimate for the cell concentrations (Venrick, 1978). Pico-phytoplankton samples were preserved with paraformaldehyde $(0.2 \%$ final concentration) and
Table 2

Abbreviations of the phytoplankton pigments analyzed in this paper.

\begin{tabular}{|c|c|}
\hline Abbreviation & Description \\
\hline \multicolumn{2}{|l|}{ Chlorophyll } \\
\hline Chl_a & Chlorophyll $a$ \\
\hline DV_Chl_a & Divinyl chlorophyll $a$ \\
\hline Chlide_a & Chlorophyllide $a$ \\
\hline TChl_a & Chl_ $a+$ DV $\_C h l \_a+$ Chlide_ $a$ \\
\hline Chl $\_b$ & Chlorophyll $b$ \\
\hline DV_Chl_ $b$ & Divinyl chlorophyll $b$ \\
\hline TChl_b & Chl $\_b+$ DV_Chl_b \\
\hline Chl_c $c_{1} / c_{2}$ & Sum of chlorophyll $c_{1}$ and $c_{2}$ \\
\hline Chl_c $c_{3}$ & Chlorophyll $c_{3}$ \\
\hline TChl_c & Chl_ $c_{1} / c_{2}+$ Chl_c $c_{3}$ \\
\hline \multicolumn{2}{|c|}{ Photosynthetic carotenoid pigments (PSC) } \\
\hline Caro & Carotene \\
\hline Fuco & Fucoxanthin \\
\hline Perid & Peridinin \\
\hline But & 19'-butanoyloxyfucoxanthin \\
\hline Hex & 19'-hexanoyloxyfucoxanthin \\
\hline \multicolumn{2}{|c|}{ Photoprotective carotenoid pigments (PPC) } \\
\hline Diad & Diadinoxanthin \\
\hline Diato & Diatoxanthin \\
\hline Dia & Diad + Diato \\
\hline Allo & Alloxanthin \\
\hline Zea & Zeaxanthin \\
\hline \multicolumn{2}{|c|}{ Tertiary pigments } \\
\hline Lut & Lutein \\
\hline Neo & Neoxanthin \\
\hline Viola & Violaxanthin \\
\hline Pras & Prasinoxanthin \\
\hline
\end{tabular}

cyanobacterial abundances were estimated by counting their picosized fraction using flow cytometry. Match-up stations with both cell counts and HPLC pigment measurements were limited to the BIOME5 cruise during 31 October 2006, CBP cruises during 11 November 2006 (CBP_5) and 3 July 2007 (CBP_8), and CliVEC-1 cruise during 17 to 28 August 2009 (Table 1 ).

\subsection{Phytoplankton taxonomy from HPLC pigments}

Phytoplankton composition was derived from the relative pigment ratios for each phytoplankton taxonomic group using CHEMTAX v1.95 (Mackey et al., 1996) and based on concentration matrices of biomarker HPLC pigments and TChl_a. The chemotaxonomy was tuned to this study region, as described below. The selected 11 phytoplankton taxonomic groups included here are: diatoms, cryptophytes, dinoflagellates containing fucoxanthin-like pigments (Dino_A), dinoflagellates containing peridinin (Dino_B), Type_8 Haptophytes defined in Zapata et al. (2004) (Hapt_A), Type_6 Haptophytes defined in Zapata et al. (2004) (Hapt_B), prasinophytes containing prasinoxanthin (Prasino_A), prasinophytes without prasinoxanthin (Prasino_B), chlorophytes, cyanobacteria, and prochlorophytes (Table 3). Euglenophytes were not considered in this study due to their relatively low abundances in this region (Marshall et al., 2006). Chrysophytes are also excluded here due to the difficulty of deriving their major accessory pigment, 19'-butanoyloxyfucoxanthin (But), from satellite ocean color (Pan et al., 2010), and the consideration that blooms of chrysophytes (e.g. Aureococcus anophagefferens) are typically limited to coastal lagoons along the U.S. northeast coast, especially in Narragansett Bay (Rhode Island), Barnegat Bay (New Jersey), and Peconics-Gardiners Bay estuary and south shores of Long Island (New York) (ECOHAB, 1994).

The distribution of [TChl_a] after logarithm transformation, log ([TChl_a]), from our pigment data set mostly (868 of 878 samples) varied between -0.9 and 1.5 . The pigment data were then separated into 12 sub-sets with $\log ([$ TChl_a]) intervals of 0.2 (Table 4). Although the separation of sub-sets is relatively 
Table 3

Phytoplankton taxonomic groups analyzed for CHEMTAX.

\begin{tabular}{|c|c|c|}
\hline Groups & Division/class & Description and example species \\
\hline $1=$ Diatom & Bacillariophyta & $\begin{array}{l}\text { Example species include Asterionellopsis } \\
\text { glacialis and Skeletonema costatum. }\end{array}$ \\
\hline $2=$ Crypt & Cryptophyta & Example species include Cryptomonas spp. \\
\hline 3= Dino $\_A$ & Dinophyta_A & $\begin{array}{l}\text { Dinoflagellates containing fucoxanthin-like pig- } \\
\text { ments(Fuco, But, or Hex), e.g. Gymnodinium spp. }\end{array}$ \\
\hline $4=$ Dino_B & Dinophyta_B & $\begin{array}{l}\text { Dinoflagellates containing peridinin, e.g. } \\
\text { Gyrodinium spp., Scrippsiella trochoidea, and } \\
\text { Heterocapsa rotundatum }\end{array}$ \\
\hline $5=$ Hapt $\_$A & Haptophyta_A & $\begin{array}{l}\text { Type_8 Haptophytes defined in Zapata et al. } \\
\text { (2004), e.g. Phaeocystis pouchetii. }\end{array}$ \\
\hline 6= Hapt $\_B$ & Haptophyta_B & $\begin{array}{l}\text { Type_6 Haptophytes defined in Zapata et al. } \\
\text { (2004), e.g. Emiliania huxleyi. }\end{array}$ \\
\hline $7=$ Prasino $\_A$ & Prasinophyceae_A & $\begin{array}{l}\text { Prasinophytes containing prasinoxanthin, e.g. } \\
\text { Pycnococcus provasolii. }\end{array}$ \\
\hline $8=$ Prasino $\_B$ & Prasinophyceae_B & $\begin{array}{l}\text { Prasinophytes without prasinoxanthin, e.g. } \\
\text { Pyramimonas grassi. }\end{array}$ \\
\hline $9=$ Chlo & Chlorophyceae & $\begin{array}{l}\text { Example species include Ankistrodesmus } \\
\text { falcatus and Nannochloris atomus. }\end{array}$ \\
\hline $10=$ Cyano & Cyanophyta & $\begin{array}{l}\text { Example species include Synechococcus spp. } \\
\text { and Unidentified Trichomes. }\end{array}$ \\
\hline $11=$ Proc & Prochlorophyta & $\begin{array}{l}\text { Example species include Prochlorococcus } \\
\text { marinus. }\end{array}$ \\
\hline
\end{tabular}

arbitrary, it is not without biological foundation. Hirata et al. (2011; Fig. 3 in their paper) indicated that as [TChl_a] increased globally the proportion of pico-phytoplankton generally decreased and micro-phytoplankton increased, and nano-phytoplankton were generally more abundant within the mid-range of [TChl_a] (e.g. 0.1 to $1 \mathrm{mg} \mathrm{m}^{-3}$ ) than in low and high [TChl_a]. Individual phytoplankton taxonomic function groups can be categorized within specific size classes, such as prochlorophytes, cyanobacteria, and pico-eukaryotes classified as pico-phytoplankton, prymnesiophytes and green algae classified as nano-phytoplankton, and diatoms and dinoflagellates classified as micro-phytoplankton. The distributions of these taxonomic groups generally followed the trends corresponding to their size class (Hirata et al., 2011). Similar trends were also observed for the northeast U.S. continental margin (Fig. 2) following the size class definitions of Vidussi et al. (2001). Such results imply that increasing the number of subsets, e.g. changing the intervals of $\log \left(\left[\mathrm{TChl} \_a\right]\right)$ to 0.1 or even 0.05 , may be beneficial for development of more appropriate pigment matrices for phytoplankton functional groups. However, the requirement to have a sufficient number of data points for each sub-set being applied to CHEMTAX, e.g. $\mathrm{N} \geq 11$, limits the number of sub-sets that we can apply to our database.

For each sub-set, the following steps adapted from the instruction manual for CHEMTAX v1.95 were followed to determine the pigment ratios. Initial pigment ratios (A00) were selected from Mackey et al. (1996). Sixty ratio matrices (A01-A60) were generated by multiplying a randomly determined factor $\left(\mathrm{M}_{\text {random }}\right)$ to each value in the initial matrix (A00), where $M_{\text {random }}=1+0.7\left(R_{\text {random }}\right)-0.5$ and $R_{\text {random }}$ is a random value between 0 and 1 generated by Excel ${ }^{\circledR}$ (Microsoft, Inc.). In total 61 solutions were created. The best $10 \%(N=6)$ of the solutions with the lowest root mean square errors (RMSEs) provided by CHEMTAX were averaged as the starting ratios $\left(\mathrm{F}_{\text {start }}\right)$. The final pigment ratios $\left(F_{n}\right)$ were then determined by CHEMTAX with the input of the starting ratios $\left(\mathrm{F}_{\text {start }}\right)$. The TChl_a concentrations for each taxonomic group $\left(\mathrm{C}_{\text {taxa_n }}\right)$ were calculated based on the final pigment ratios $\left(\mathrm{F}_{\mathrm{n}}\right)$. Although DV_Chl_a, But, Hex, and Pras were applied to construct the pigment concentration matrices, they were not used to interpret or further applied to satellite imagery due to the difficulty in retrieving those pigment signatures from ocean color remote sensing data (Pan et al., 2010). The ratios for TChl_c represent the sum of Chl $\_c_{1} / c_{2}$ and $C h l_{-} c_{3}$. Individual pigment concentrations
([Pig_m]) integrated from all taxonomic groups were calculated with Eq. (1).

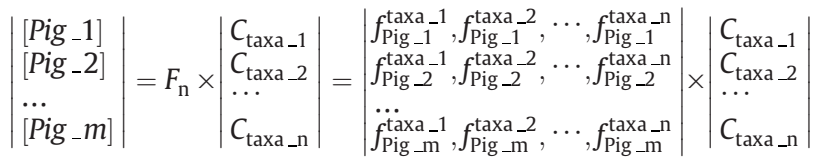

Here, Pig_m represents individual HPLC pigment type including TChl_a, TChl_b, TChl_c, Fuco, Perid, Zea, Allo, Dia, Lut, Neo, and Viola, and $f_{\text {Pig } \_m}^{\text {taxa } n}$ in the $n \times m$ (here $11 \times 11$ ) matrices $\left(F_{n}\right)$ represents the mth marker pigment ratio normalized to $C_{\text {taxa_n }}$ for the nth phytoplankton taxa, and $C_{\text {taxa_n }}$ represents the relevant TChl_ $a$ for the nth phytoplankton functional group. The fraction of TChl_a for each phytoplankton taxonomic group [(FC) $\left.)_{\text {taxa_n }}\right]$ is then calculated as:

$(F C)_{\text {taxa_n }}=\frac{C_{\text {taxa } \_n}}{[\text { TChl_a }]} \times 100 \%$.

Ideally, the reconstructed pigment concentrations ([Pig_m $]_{\text {reconstructed }}$ ) computed by multiplying CHEMTAX-derived concentrations of phytoplankton functional groups $\left(\mathrm{C}_{\text {taxa_n }}\right)$ with the pigment ratio matrices $\left(F_{n}\right)$ should be equal to the field measurements of HPLC pigments ([Pig_m $\left.]_{\text {field }}\right)$. The difference between [Pig_m $]_{\text {reconstructed }}$ and [Pig_m $]_{\text {field }}$ was then considered as the deviations caused by the CHEMTAX method. The mean absolute percent difference (MAPD) and root mean square error (RMSE) between reconstructed and field pigment concentrations were defined as:

$$
(M A P D)_{\text {Pig_m }}=\frac{1}{N} \sum \frac{\mid[\text { Pig_m } m]_{\text {reconstructed }}-[\text { Pig_m } m]_{\text {field }} \mid}{[\text { Pig_m }]_{\text {field }}} \times 100 \%
$$

$(R M S E)_{\text {Pig_m }}=\sqrt{\frac{1}{N} \sum\left([\text { Pig_m }]_{\text {reconstructed }}-[\text { Pig_m }]_{\text {field }}\right)^{2}}$.

Here, $\mathrm{N}$ represents the number of samples.

\subsection{Satellite imagery}

Observations of level-2 spectral remote sensing reflectance $\left(R_{r s}\right)$ from the Moderate Resolution Imaging Spectroradiometer on the Aqua satellite (MODIS-Aqua) were downloaded from the NASA Ocean Color Web (http://oceancolor.gsfc.nasa.gov/; by access during 19 to 25 November 2010). Bulk water temperature required to estimate [Zea] (Pan et al., 2010) was obtained from MODIS-Aqua shortwave infrared ( $4 \mu \mathrm{m}$ ) observations of nighttime sea surface temperature (SST). All images were mapped isotropically to 90 pixels per longitude degree and 111 pixels per latitude degree (with an approximate resolution of $1 \mathrm{~km} \times 1 \mathrm{~km}$ per pixel) using the SeaWiFS Data Analysis System software (SeaDAS; version 6.1).

Phytoplankton pigment concentrations ([Pig_m]) were calculated from the empirical algorithms based on the $\mathrm{R}_{\mathrm{rs}}$ band ratios as described by Pan et al. (2010):

$\log [$ Pig_m $]=A_{0, \mathrm{~m}}+A_{1, \mathrm{~m}} X+A_{2, \mathrm{~m}} X^{2}+A_{3, \mathrm{~m}} X^{3}$.

Here $X=\log \left[R_{r s}\left(\lambda_{1}\right) / R_{r s}\left(\lambda_{2}\right)\right]$, and $A_{i, m}(i=0,1,2$, and 3) were derived coefficients for each pigment. The selected MODIS bands were $488 \mathrm{~nm}$ for $\lambda_{1}$ and 555 or $667 \mathrm{~nm}$ for $\lambda_{2}$ (Pan et al., 2010).

$\mathrm{C}_{\text {taxa_n }}$ was then determined by solving Eq. (1) through multiple linear regressions with the derived $F_{n}$ and [Pig_m]. The multiple 
Table 4

Final pigment matrices of phytoplankton taxonomic groups, $f_{\text {Pig_m }}^{\text {taxa }}$, derived from CHEMTAX for 12 sub-sets based on log([TChl_a]) distributions.

\begin{tabular}{|c|c|c|c|c|c|c|c|c|c|c|c|}
\hline Groups & TChl_a & TChl_b & TChl_c & Perid & Fuco & Dia & Allo & Zea & Lut & Neo & Viola \\
\hline \multicolumn{12}{|c|}{ Sub-set 1: $\log ([$ TChl_a $])<-0.7$} \\
\hline Diatom & 1 & 0 & 0.2108 & 0 & 0.7552 & 0.0626 & 0 & 0 & 0 & 0 & 0 \\
\hline Crypt & 1 & 0 & 0.0797 & 0 & 0 & 0 & 0.3655 & 0 & 0 & 0 & 0 \\
\hline Dino_A & 1 & 0 & 0.2244 & 0 & 0.4762 & 0.7847 & 0 & 0 & 0 & 0 & 0 \\
\hline Dino_B & 1 & 0 & 0.3844 & 0.5903 & 0 & 0.1683 & 0 & 0 & 0 & 0 & 0 \\
\hline Hapt_A & 1 & 0 & 0.5139 & 0 & 0.4319 & 0.0508 & 0 & 0 & 0 & 0 & 0 \\
\hline Hapt_B & 1 & 0 & 0.3569 & 0 & 0.2368 & 0.2744 & 0 & 0 & 0 & 0 & 0 \\
\hline Prasino_A & 1 & 0.8437 & 0 & 0 & 0 & 0 & 0 & 0.0828 & 0.0198 & 0.1013 & 0.0074 \\
\hline Prasino_B & 1 & 1.1130 & 0 & 0 & 0 & 0 & 0 & 0.0636 & 0.0657 & 0.1154 & 0.1180 \\
\hline Chlo & 1 & 0.0263 & 0 & 0 & 0 & 0 & 0 & 0.0239 & 0.0260 & 0.0266 & 0.0202 \\
\hline Cyano & 1 & 0 & 0 & 0 & 0 & 0 & 0 & 1.1889 & 0 & 0 & 0 \\
\hline Proc & 1 & 0.3552 & 0 & 0 & 0 & 0 & 0 & 0.2320 & 0 & 0 & 0 \\
\hline \multicolumn{12}{|c|}{ Sub-set $2:-0.7 \leq \log ([$ TChl_a $])<-0.5$} \\
\hline Diatom & 1 & 0 & 0.2312 & 0 & 0.5793 & 0.0663 & 0 & 0 & 0 & 0 & 0 \\
\hline Crypt & 1 & 0 & 0.0723 & 0 & 0 & 0 & 0.3289 & 0 & 0 & 0 & 0 \\
\hline Dino_A & 1 & 0 & 0.2136 & 0 & 0.4913 & 0.8545 & 0 & 0 & 0 & 0 & 0 \\
\hline Dino_B & 1 & 0 & 0.4166 & 0.6195 & 0 & 0.2245 & 0 & 0 & 0 & 0 & 0 \\
\hline Hapt_A & 1 & 0 & 0.1928 & 0 & 0.2077 & 0.0264 & 0 & 0 & 0 & 0 & 0 \\
\hline Hapt_B & 1 & 0 & 0.3922 & 0 & 0.1194 & 0.2933 & 0 & 0 & 0 & 0 & 0 \\
\hline Prasino_A & 1 & 0.7173 & 0 & 0 & 0 & 0 & 0 & 0.0696 & 0.0186 & 0.0792 & 0.0087 \\
\hline Prasino_B & 1 & 0.5602 & 0 & 0 & 0 & 0 & 0 & 0.0476 & 0.0480 & 0.0895 & 0.1126 \\
\hline Chlo & 1 & 0.3911 & 0 & 0 & 0 & 0 & 0 & 0.0532 & 0.1918 & 0.0446 & 0.0392 \\
\hline Cyano & 1 & 0 & 0 & 0 & 0 & 0 & 0 & 0.9459 & 0 & 0 & 0 \\
\hline Proc & 1 & 0.5536 & 0 & 0 & 0 & 0 & 0 & 0.2410 & 0 & 0 & 0 \\
\hline \multicolumn{12}{|c|}{ Sub-set 3: $-0.5 \leq \log ([$ TChl_a $])<-0.3$} \\
\hline Diatom & 1 & 0 & 0.2097 & 0 & 0.6930 & 0.0558 & 0 & 0 & 0 & 0 & 0 \\
\hline Crypt & 1 & 0 & 0.0658 & 0 & 0 & 0 & 0.3039 & 0 & 0 & 0 & 0 \\
\hline Dino_A & 1 & 0 & 0.1699 & 0 & 0.5120 & 0.9437 & 0 & 0 & 0 & 0 & 0 \\
\hline Dino_B & 1 & 0 & 0.4565 & 0.3654 & 0 & 0.1807 & 0 & 0 & 0 & 0 & 0 \\
\hline Hapt_A & 1 & 0 & 0.3533 & 0 & 0.3150 & 0.0396 & 0 & 0 & 0 & 0 & 0 \\
\hline Hapt_B & 1 & 0 & 0.3508 & 0 & 0.1675 & 0.3236 & 0 & 0 & 0 & 0 & 0 \\
\hline Prasino_A & 1 & 0.6809 & 0 & 0 & 0 & 0 & 0 & 0.0842 & 0.0173 & 0.1013 & 0.0098 \\
\hline Prasino_B & 1 & 0.8977 & 0 & 0 & 0 & 0 & 0 & 0.0474 & 0.0675 & 0.0973 & 0.1223 \\
\hline Chlo & 1 & 0.2432 & 0 & 0 & 0 & 0 & 0 & 0.0410 & 0.1771 & 0.0453 & 0.0306 \\
\hline Cyano & 1 & 0 & 0 & 0 & 0 & 0 & 0 & 0.8097 & 0 & 0 & 0 \\
\hline Proc & 1 & 0.6461 & 0 & 0 & 0 & 0 & 0 & 0.2328 & 0 & 0 & 0 \\
\hline \multicolumn{12}{|c|}{ Sub-set 4: $-0.3 \leq \log ([$ TChl_a $])<-0.1$} \\
\hline Diatom & 1 & 0 & 0.1770 & 0 & 0.5820 & 0.0603 & 0 & 0 & 0 & 0 & 0 \\
\hline Crypt & 1 & 0 & 0.0696 & 0 & 0 & 0 & 0.2628 & 0 & 0 & 0 & 0 \\
\hline Dino_A & 1 & 0 & 0.1862 & 0 & 0.5473 & 0.8401 & 0 & 0 & 0 & 0 & 0 \\
\hline Dino_B & 1 & 0 & 0.3457 & 0.4980 & 0 & 0.1456 & 0 & 0 & 0 & 0 & 0 \\
\hline Hapt_A & 1 & 0 & 0.4411 & 0 & 0.2174 & 0.0348 & 0 & 0 & 0 & 0 & 0 \\
\hline Hapt_B & 1 & 0 & 0.3611 & 0 & 0.2657 & 0.2484 & 0 & 0 & 0 & 0 & 0 \\
\hline Prasino_A & 1 & 0.7503 & 0 & 0 & 0 & 0 & 0 & 0.0896 & 0.0174 & 0.0966 & 0.0089 \\
\hline Prasino_B & 1 & 0.9162 & 0 & 0 & 0 & 0 & 0 & 0.0556 & 0.0624 & 0.0973 & 0.1017 \\
\hline Chlo & 1 & 0.2858 & 0 & 0 & 0 & 0 & 0 & 0.0389 & 0.1466 & 0.0468 & 0.0317 \\
\hline Cyano & 1 & 0 & 0 & 0 & 0 & 0 & 0 & 0.6017 & 0 & 0 & 0 \\
\hline Proc & 1 & 0.5071 & 0 & 0 & 0 & 0 & 0 & 0.2126 & 0 & 0 & 0 \\
\hline \multicolumn{12}{|c|}{ Sub-set 5: $-0.1 \leq \log ([$ TChl_a $])<0.1$} \\
\hline Diatom & 1 & 0 & 0.1852 & 0 & 0.6342 & 0.0561 & 0 & 0 & 0 & 0 & 0 \\
\hline Crypt & 1 & 0 & 0.0634 & 0 & 0 & 0 & 0.2458 & 0 & 0 & 0 & 0 \\
\hline Dino_A & 1 & 0 & 0.1948 & 0 & 0.4429 & 0.6610 & 0 & 0 & 0 & 0 & 0 \\
\hline Dino_B & 1 & 0 & 0.3334 & 0.7643 & 0 & 0.1726 & 0 & 0 & 0 & 0 & 0 \\
\hline Hapt_A & 1 & 0 & 0.4957 & 0 & 0.2595 & 0.0467 & 0 & 0 & 0 & 0 & 0 \\
\hline Hapt_B & 1 & 0 & 0.3879 & 0 & 0.2099 & 0.1156 & 0 & 0 & 0 & 0 & 0 \\
\hline Prasino_A & 1 & 0.7244 & 0 & 0 & 0 & 0 & 0 & 0.0642 & 0.0165 & 0.0916 & 0.0085 \\
\hline Prasino_B & 1 & 0.8759 & 0 & 0 & 0 & 0 & 0 & 0.0546 & 0.0556 & 0.0881 & 0.1209 \\
\hline Chlo & 1 & 0.2350 & 0 & 0 & 0 & 0 & 0 & 0.0360 & 0.1690 & 0.0388 & 0.0285 \\
\hline Cyano & 1 & 0 & 0 & 0 & 0 & 0 & 0 & 0.9549 & 0 & 0 & 0 \\
\hline Proc & 1 & 0.6052 & 0 & 0 & 0 & 0 & 0 & 0.2337 & 0 & 0 & 0 \\
\hline \multicolumn{12}{|c|}{ Sub-set 6: $0.1 \leq \log ([$ TChl_a $])<0.3$} \\
\hline Diatom & 1 & 0 & 0.1606 & 0 & 0.6178 & 0.0510 & 0 & 0 & 0 & 0 & 0 \\
\hline Crypt & 1 & 0 & 0.0837 & 0 & 0 & 0 & 0.2115 & 0 & 0 & 0 & 0 \\
\hline Dino_A & 1 & 0 & 0.2351 & 0 & 0.4779 & 0.8808 & 0 & 0 & 0 & 0 & 0 \\
\hline Dino_B & 1 & 0 & 0.3021 & 0.7737 & 0 & 0.1378 & 0 & 0 & 0 & 0 & 0 \\
\hline Hapt_A & 1 & 0 & 0.8328 & 0 & 0.4125 & 0.0527 & 0 & 0 & 0 & 0 & 0 \\
\hline Hapt_B & 1 & 0 & 0.3498 & 0 & 0.2336 & 0.0950 & 0 & 0 & 0 & 0 & 0 \\
\hline Prasino_A & 1 & 0.7738 & 0 & 0 & 0 & 0 & 0 & 0.0750 & 0.0178 & 0.0900 & 0.0086 \\
\hline Prasino_B & 1 & 0.8782 & 0 & 0 & 0 & 0 & 0 & 0.0447 & 0.0435 & 0.0913 & 0.1044 \\
\hline
\end{tabular}


Table 4 (continued)

\begin{tabular}{|c|c|c|c|c|c|c|c|c|c|c|c|}
\hline Groups & TChl_a & TChl_b & TChl_c & Perid & Fuco & Dia & Allo & Zea & Lut & Neo & Viola \\
\hline \multicolumn{12}{|c|}{ Sub-set 6: $0.1 \leq \log ([$ TChl_a $])<0.3$} \\
\hline Chlo & 1 & 0.2165 & 0 & 0 & 0 & 0 & 0 & 0.0277 & 0.1057 & 0.0295 & 0.0259 \\
\hline Cyano & 1 & 0 & 0 & 0 & 0 & 0 & 0 & 0.7079 & 0 & 0 & 0 \\
\hline Proc & 1 & 0.5547 & 0 & 0 & 0 & 0 & 0 & 0.2310 & 0 & 0 & 0 \\
\hline \multicolumn{12}{|c|}{ Sub-set 7: $0.3 \leq \log ([$ TChl_a] $)<0.5$} \\
\hline Diatom & 1 & 0 & 0.1696 & 0 & 0.5458 & 0.0438 & 0 & 0 & 0 & 0 & 0 \\
\hline Crypt & 1 & 0 & 0.0904 & 0 & 0 & 0 & 0.2366 & 0 & 0 & 0 & 0 \\
\hline Dino_A & 1 & 0 & 0.1729 & 0 & 0.3969 & 0.8260 & 0 & 0 & 0 & 0 & 0 \\
\hline Dino_B & 1 & 0 & 0.3003 & 0.8031 & 0 & 0.1340 & 0 & 0 & 0 & 0 & 0 \\
\hline Hapt_A & 1 & 0 & 0.6823 & 0 & 0.6388 & 0.0621 & 0 & 0 & 0 & 0 & 0 \\
\hline Hapt_B & 1 & 0 & 0.4305 & 0 & 0.2548 & 0.2509 & 0 & 0 & 0 & 0 & 0 \\
\hline Prasino_A & 1 & 0.6680 & 0 & 0 & 0 & 0 & 0 & 0.0659 & 0.0168 & 0.0718 & 0.0076 \\
\hline Prasino_B & 1 & 0.7792 & 0 & 0 & 0 & 0 & 0 & 0.0527 & 0.0511 & 0.0936 & 0.0972 \\
\hline Chlo & 1 & 0.2347 & 0 & 0 & 0 & 0 & 0 & 0.0293 & 0.0519 & 0.0289 & 0.0269 \\
\hline Cyano & 1 & 0 & 0 & 0 & 0 & 0 & 0 & 0.7114 & 0 & 0 & 0 \\
\hline Proc & 1 & 0.5923 & 0 & 0 & 0 & 0 & 0 & 0.2354 & 0 & 0 & 0 \\
\hline \multicolumn{12}{|c|}{ Sub-set 8: $0.5 \leq \log ([$ TChl_a $])<0.7$} \\
\hline Diatom & 1 & 0 & 0.1576 & 0 & 0.5122 & 0.0379 & 0 & 0 & 0 & 0 & 0 \\
\hline Crypt & 1 & 0 & 0.0918 & 0 & 0 & 0 & 0.2572 & 0 & 0 & 0 & 0 \\
\hline Dino_A & 1 & 0 & 0.1826 & 0 & 0.4009 & 0.8294 & 0 & 0 & 0 & 0 & 0 \\
\hline Dino_B & 1 & 0 & 0.2655 & 0.7556 & 0 & 0.1188 & 0 & 0 & 0 & 0 & 0 \\
\hline Hapt_A & 1 & 0 & 1.0504 & 0 & 0.5077 & 0.0633 & 0 & 0 & 0 & 0 & 0 \\
\hline Hapt_B & 1 & 0 & 0.3989 & 0 & 0.2220 & 0.1146 & 0 & 0 & 0 & 0 & 0 \\
\hline Prasino_A & 1 & 0.7411 & 0 & 0 & 0 & 0 & 0 & 0.0692 & 0.0140 & 0.0747 & 0.0083 \\
\hline Prasino_B & 1 & 0.7644 & 0 & 0 & 0 & 0 & 0 & 0.0454 & 0.0534 & 0.0811 & 0.0945 \\
\hline Chlo & 1 & 0.2374 & 0 & 0 & 0 & 0 & 0 & 0.0282 & 0.1165 & 0.0320 & 0.0250 \\
\hline Cyano & 1 & 0 & 0 & 0 & 0 & 0 & 0 & 0.6473 & 0 & 0 & 0 \\
\hline Proc & 1 & 0.6029 & 0 & 0 & 0 & 0 & 0 & 0.1941 & 0 & 0 & 0 \\
\hline \multicolumn{12}{|c|}{ Sub-set 9: $0.7 \leq \log ([$ TChl_a $])<0.9$} \\
\hline Diatom & 1 & 0 & 0.1603 & 0 & 0.5176 & 0.0347 & 0 & 0 & 0 & 0 & 0 \\
\hline Crypt & 1 & 0 & 0.0691 & 0 & 0 & 0 & 0.3008 & 0 & 0 & 0 & 0 \\
\hline Dino_A & 1 & 0 & 0.2056 & 0 & 0.5969 & 0.9267 & 0 & 0 & 0 & 0 & 0 \\
\hline Dino_B & 1 & 0 & 0.3022 & 0.7063 & 0 & 0.1776 & 0 & 0 & 0 & 0 & 0 \\
\hline Hapt_A & 1 & 0 & 0.6087 & 0 & 0.3062 & 0.0401 & 0 & 0 & 0 & 0 & 0 \\
\hline Hapt_B & 1 & 0 & 0.4735 & 0 & 0.2654 & 0.3018 & 0 & 0 & 0 & 0 & 0 \\
\hline Prasino_A & 1 & 0.8318 & 0 & 0 & 0 & 0 & 0 & 0.0839 & 0.0176 & 0.0876 & 0.0078 \\
\hline Prasino_B & 1 & 0.8019 & 0 & 0 & 0 & 0 & 0 & 0.0467 & 0.0491 & 0.0837 & 0.1033 \\
\hline Chlo & 1 & 0.1475 & 0 & 0 & 0 & 0 & 0 & 0.0242 & 0.0181 & 0.0249 & 0.0217 \\
\hline Cyano & 1 & 0 & 0 & 0 & 0 & 0 & 0 & 0.6056 & 0 & 0 & 0 \\
\hline Proc & 1 & 0.6283 & 0 & 0 & 0 & 0 & 0 & 0.2644 & 0 & 0 & 0 \\
\hline \multicolumn{12}{|c|}{ Sub-set $10: 0.9 \leq \log ([$ TChl_a] $)<1.1$} \\
\hline Diatom & 1 & 0 & 0.1483 & 0 & 0.5106 & 0.0342 & 0 & 0 & 0 & 0 & 0 \\
\hline Crypt & 1 & 0 & 0.0738 & 0 & 0 & 0 & 0.2765 & 0 & 0 & 0 & 0 \\
\hline Dino_A & 1 & 0 & 0.1270 & 0 & 0.5244 & 0.8351 & 0 & 0 & 0 & 0 & 0 \\
\hline Dino_B & 1 & 0 & 0.2847 & 0.7034 & 0 & 0.1716 & 0 & 0 & 0 & 0 & 0 \\
\hline Hapt_A & 1 & 0 & 0.8304 & 0 & 0.3343 & 0.0473 & 0 & 0 & 0 & 0 & 0 \\
\hline Hapt_B & 1 & 0 & 0.4412 & 0 & 0.2926 & 0.3269 & 0 & 0 & 0 & 0 & 0 \\
\hline Prasino_A & 1 & 0.9292 & 0 & 0 & 0 & 0 & 0 & 0.0967 & 0.0185 & 0.1134 & 0.0100 \\
\hline Prasino_B & 1 & 0.8432 & 0 & 0 & 0 & 0 & 0 & 0.0517 & 0.0602 & 0.0826 & 0.1002 \\
\hline Chlo & 1 & 0.1097 & 0 & 0 & 0 & 0 & 0 & 0.0223 & 0.0099 & 0.0213 & 0.0165 \\
\hline Cyano & 1 & 0 & 0 & 0 & 0 & 0 & 0 & 0.8287 & 0 & 0 & 0 \\
\hline Proc & 1 & 0.6819 & 0 & 0 & 0 & 0 & 0 & 0.2507 & 0 & 0 & 0 \\
\hline \multicolumn{12}{|c|}{ Sub-set $11: 1.1 \leq \log ([$ TChl_a] $)<1.3$} \\
\hline Diatom & 1 & 0 & 0.1398 & 0 & 0.4607 & 0.0427 & 0 & 0 & 0 & 0 & 0 \\
\hline Crypt & 1 & 0 & 0.0747 & 0 & 0 & 0 & 0.2897 & 0 & 0 & 0 & 0 \\
\hline Dino_A & 1 & 0 & 0.1716 & 0 & 0.3616 & 1.0481 & 0 & 0 & 0 & 0 & 0 \\
\hline Dino_B & 1 & 0 & 0.2508 & 0.6579 & 0 & 0.1476 & 0 & 0 & 0 & 0 & 0 \\
\hline Hapt_A & 1 & 0 & 0.6767 & 0 & 0.3641 & 0.0505 & 0 & 0 & 0 & 0 & 0 \\
\hline Hapt_B & 1 & 0 & 0.4435 & 0 & 0.1868 & 0.1550 & 0 & 0 & 0 & 0 & 0 \\
\hline Prasino_A & 1 & 0.8959 & 0 & 0 & 0 & 0 & 0 & 0.1036 & 0.0156 & 0.1207 & 0.0101 \\
\hline Prasino_B & 1 & 0.7231 & 0 & 0 & 0 & 0 & 0 & 0.0452 & 0.0478 & 0.0851 & 0.0989 \\
\hline Chlo & 1 & 0.3226 & 0 & 0 & 0 & 0 & 0 & 0.0429 & 0.1474 & 0.0468 & 0.0361 \\
\hline Cyano & 1 & 0 & 0 & 0 & 0 & 0 & 0 & 0.6840 & 0 & 0 & 0 \\
\hline Proc & 1 & 0.6079 & 0 & 0 & 0 & 0 & 0 & 0.2312 & 0 & 0 & 0 \\
\hline \multicolumn{12}{|c|}{ Sub-set 12: $1.3 \leq \log \left(\left[T C h l \_a\right]\right)$} \\
\hline Diatom & 1 & 0 & 0.1519 & 0 & 0.4486 & 0.0765 & 0 & 0 & 0 & 0 & 0 \\
\hline Crypt & 1 & 0 & 0.1182 & 0 & 0 & 0 & 0.2291 & 0 & 0 & 0 & 0 \\
\hline Dino_A & 1 & 0 & 0.1430 & 0 & 1.1550 & 0.4188 & 0 & 0 & 0 & 0 & 0 \\
\hline Dino_B & 1 & 0 & 0.2980 & 0.7600 & 0 & 0.1482 & 0 & 0 & 0 & 0 & 0 \\
\hline Hapt_A & 1 & 0 & 0.5690 & 0 & 0.3641 & 0.0506 & 0 & 0 & 0 & 0 & 0 \\
\hline Hapt_B & 1 & 0 & 0.2901 & 0 & 0.4100 & 0.2624 & 0 & 0 & 0 & 0 & 0 \\
\hline
\end{tabular}


Table 4 (continued)

\begin{tabular}{|c|c|c|c|c|c|c|c|c|c|c|c|}
\hline Groups & TChl_a & TChl_b & TChl_c & Perid & Fuco & Dia & Allo & Zea & Lut & $\mathrm{Neo}$ & Viola \\
\hline \multicolumn{12}{|c|}{ Sub-set 12: $1.3 \leq \log \left(\left[T C h l \_a\right]\right)$} \\
\hline Prasino_A & 1 & 0.8314 & 0 & 0 & 0 & 0 & 0 & 0.0755 & 0.0205 & 0.0775 & 0.0084 \\
\hline Prasino_B & 1 & 0.4449 & 0 & 0 & 0 & 0 & 0 & 0.0335 & 0.0307 & 0.0705 & 0.0914 \\
\hline Chlo & 1 & 0.3532 & 0 & 0 & 0 & 0 & 0 & 0.0485 & 0.1580 & 0.0397 & 0.0375 \\
\hline Cyano & 1 & 0 & 0 & 0 & 0 & 0 & 0 & 0.6043 & 0 & 0 & 0 \\
\hline Proc & 1 & 0.7109 & 0 & 0 & 0 & 0 & 0 & 0.2588 & 0 & 0 & 0 \\
\hline
\end{tabular}

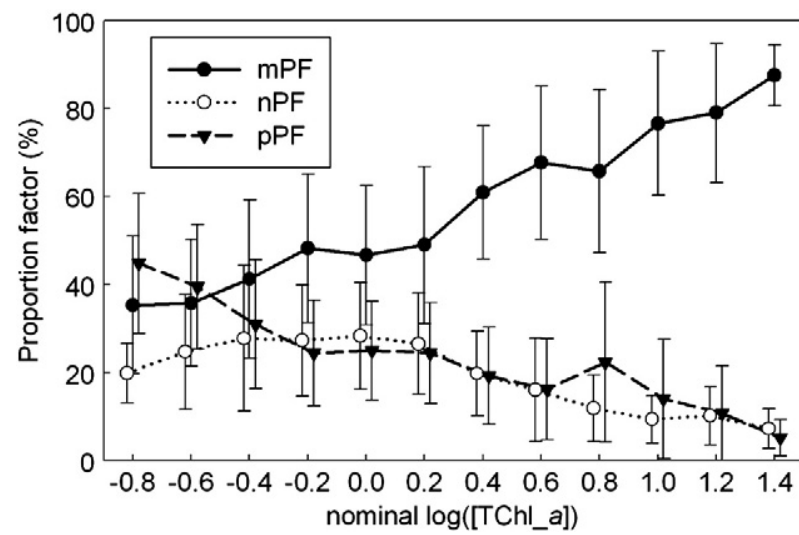

Fig. 2. The mean ( $\pm \mathrm{SD}$ ) proportion factors of micro- $(\mathrm{mPF})$, nano- $(\mathrm{nPF})$, and picophytoplankton (pPF) based on Vidussi et al. (2001) along the nominal log([TChl_a]) $($ mean \pm 0.1$)$. The definitions are: $m P F=([$ Fuco $]+[$ Perid $]) / D P, n P F=([$ Hex $]+[$ But $]+$ $[$ Allo $]) / D P$, and $p P F=([$ Zea $]+[$ TChl_b $]) / D P$, while DP $=[$ Fuco $]+[$ Perid $]+[$ Hex $]+[$ But $]+$ $[A l l o]+[Z e a]+\left[T C h l_{-} b\right]$. For a better view, the $\mathrm{x}$-axes of the corresponding $\mathrm{nPF}$ and $\mathrm{pPF}$ were artificially moved slightly left and right, respectively.

linear regression of Eq. (1) requires $m \geq n$, and in this paper, $m=n=11$. The multiple linear fit was conducted by adapting an IDL program, 'regress2.pro', which can be downloaded from http:// hesperia.gsfc.nasa.gov/ssw/gen/idl/fitting/. Above all, the methodology to derive phytoplankton community composition from space is also shown on a flowchart (Fig. 3).

\section{Results}

\subsection{CHEMTAX relative pigment concentration ratios}

In this study, we separated HPLC pigment data into 12 sub-sets based on the distributions of $\log \left(\left[\mathrm{TCh} \_a\right]\right)$. Such a separation is based on the previously observed seasonal and regional variations of dominant phytoplankton species in this study region (Marshall, 1984a; Marshall \& Cohn, 1987; Marshall et al., 2006) associating with the distributions of phytoplankton pigments (Pan et al., 2010) and the trends of the variations of phytoplankton community composition (micro-, nano-, and pico-phytoplankton) with [TChl_a] (Fig. 2; and also Hirata et al., 2011). The differences in the relative pigment concentration ratios for the phytoplankton taxonomic groups $\left(f_{\text {Pig_m }}^{\text {taxa }}\right.$ n $)$ in 12 sub-sets are shown in Table 4. $f_{\mathrm{Pig} \mathrm{m}}^{\mathrm{taxa} n}$ from the major phytoplankton types (e.g. Fuco in diatoms, Allo in cryptophytes, and Zea in cyanobacteria) generally decreases with the increase of [TChl_a], but the trends become less significant or even reverse in some less abundant phytoplankton types (e.g. Perid in Dino_B) (Table 4 and Fig. 4). $f_{\text {Pig_m }}^{\text {taxa } n}$ was less variable at relatively high [TChl_a], e.g. log ([TChl_a]) $>0$, which might indicate some dominant phytoplankton (e.g. diatoms) became more significant. In contrast, the highly variable

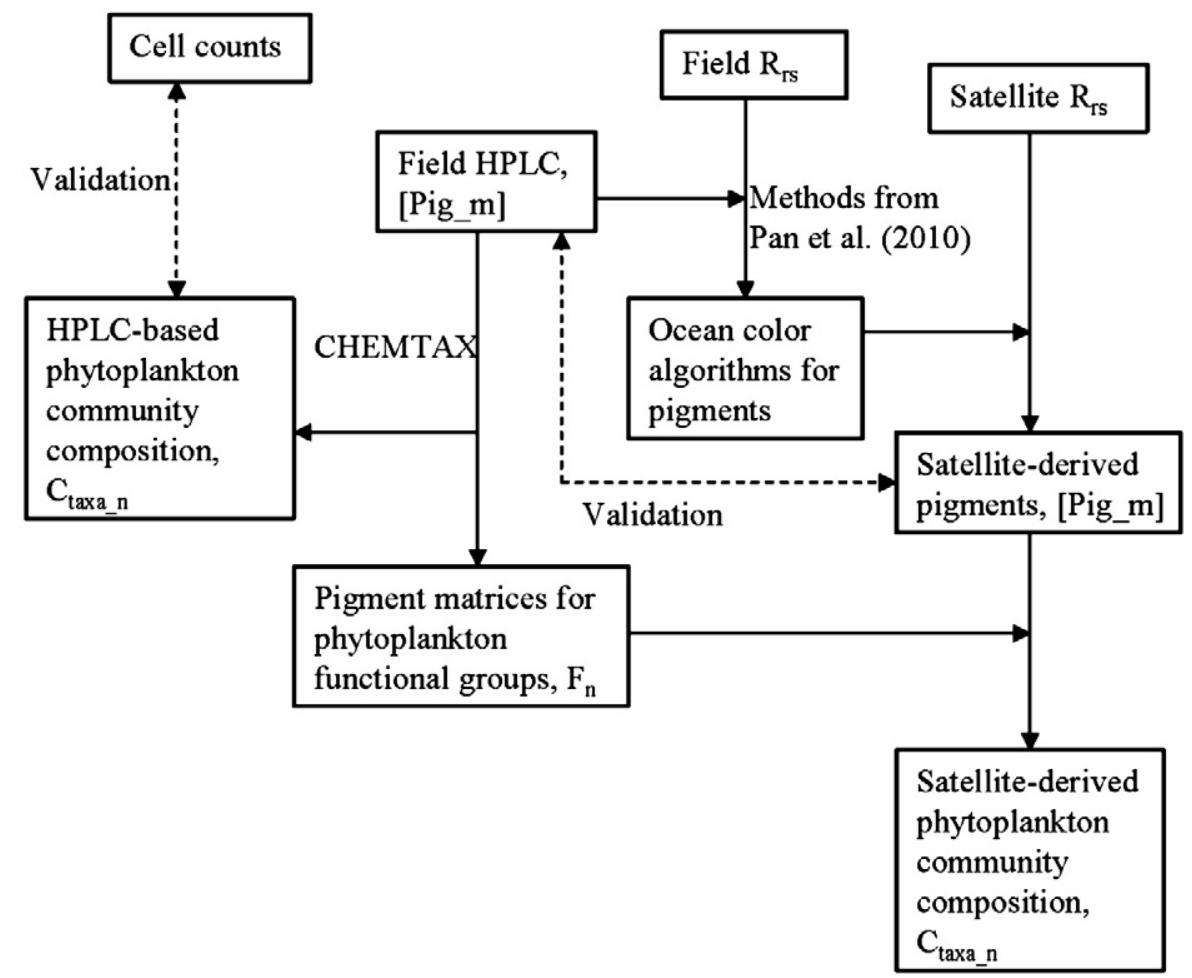

Fig. 3. Flowchart of the methodology. 


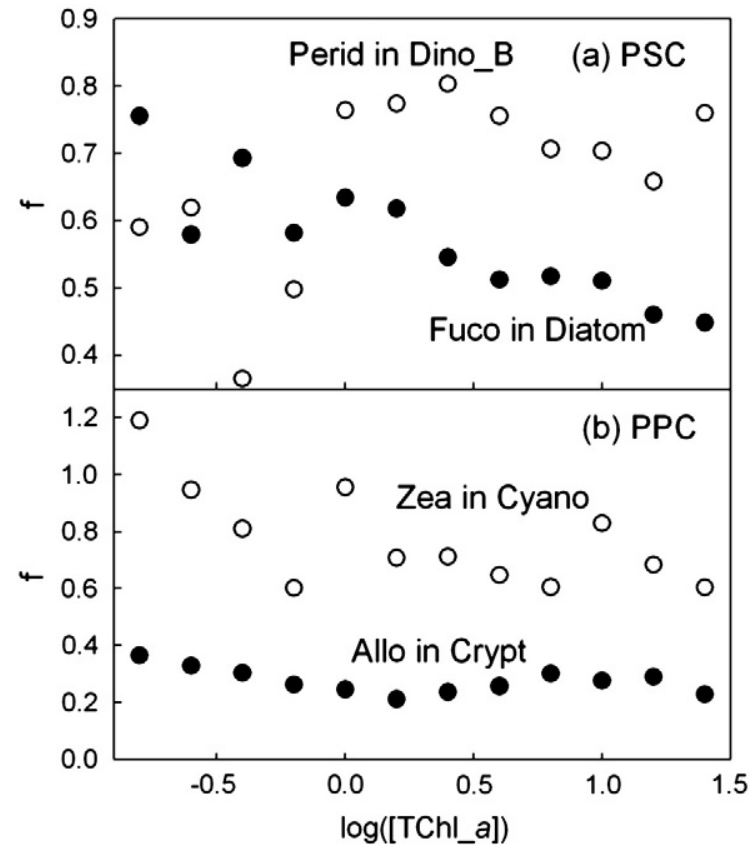

Fig. 4. Relationships between the CHEMTAX-derived relative pigment concentration ratios for the phytoplankton taxonomic groups $\left(f_{\text {Pig } \_ \text {m }}^{\text {taxan }}\right)$ and [TChl_a]. (a) Examples of photosynthetic carotenoid pigments (PSC) such as fucoxanthin (Fuco) in diatoms and peridinin (Perid) in dinoflagellates containing Perid (Dino_B); (b) Examples of photoprotective carotenoid pigments (PPC) such as alloxanthin (Allo) in cryptophytes (Crypt) and zeaxanthin (Zea) in cyanobacteria (Cyano).

$f_{\text {Pig_m }}^{\text {taxa } n}$ at relatively low $[$ TChl_a], e.g. $\log ([$ TChl_a $])<-0.3$, might suggest shifts in the dominant phytoplankton over space and time.

The CHEMTAX-derived pigment ratio matrices were generally reasonable based on the assessment of reconstructed pigment concentrations from field measurements. The mean absolute percent differences (MAPDs) of the reconstructed pigment concentrations and field measurements were generally low (4\% to $9 \%$ ) for pigments well correlated with TChl_a (e.g. Fuco, TChl_c, and Dia, which were also abundant in the most common phytoplankton species in this region, diatoms; Pan et al., 2010) (Table 5). Moderate MAPDs were found for pigments unique to phytoplankton functional groups whose abundances were generally high, e.g. $17 \%$ for Perid, $20 \%$ for Allo, and $17 \%$ for Zea (Table 5). Very high MAPDs were generally found for pigments unique to phytoplankton functional groups whose abundances were generally very low, e.g. Pras and DV_Chl_a (unpublished data). The MAPDs for the accessory pigments (TChl_b, Lut, Neo, and Viola) abundant in green algae (prasinophytes and other chlorophytes) increased with a decrease in their relative abundance, e.g. the MAPDs of TChl $\_b<\mathrm{Neo} \approx$ Viola $<$ Lut as the median pigment concentration values were [TChl_b] $>$ $[$ Neo] $\approx[$ Viola] $>$ [Lut] (Table 5). Similar trends to the MAPDs were also found for the root mean square errors (RMSEs) between the reconstructed pigment concentrations and the field measurements, after accounting for the median value in each pigment component (Table 5).

\subsection{Phytoplankton composition from field measurements}

The mean (FC) $)_{\text {taxa_n }}$ for different sub-sets are shown in Fig. 5. Diatoms generally became more abundant and dominant with the increase of [TChl_a], accounting for $17-26 \%, 24-53 \%$, and $49-70 \%$ of TChl_ $a$ at sub-sets with $\log ([$ TChl_a] $)<-0.1$, between -0.1 and 0.5 , and $>0.5$, respectively. The relative abundance of cryptophytes also increased with the increase of [TChl_a] at relatively low log ([TChl_a]) condition (e.g. $<0.3$ ), but decreased at relatively high log ([TChl_a]) condition (e.g. $>0.3$ ) primarily due to the obscuration caused by diatoms. The relative abundance of dinoflagellates (Dino_A + Dino_B) had a similar trend with cryptophytes, but the decrease of (FC) $)_{\text {Dino }}$ with [TChl_a] began earlier than cryptophytes at log $([$ TChl_a] $)=-0.3$. Haptophytes (Hapt_A + Hapt_B) generally became less abundant with the increase of [TChl_a], ranging from 19$30 \%, 6-24 \%$, and $1-5 \%$ at sub-sets with $\log ([$ TChl_a] $)<-0.1$, between -0.1 and 0.5 , and $>0.5$, respectively. The relative abundance of green algae (Prasino_A+Prasino_B + Chlo) was less variable at most [TChl_a] conditions, ranging from $9-15 \%$ at $\log \left(\left[\mathrm{TCh} \_a\right]\right)$ varying from -0.7 to 1.1. The relative abundances of cyanobacteria and prochlorophytes generally decreased with the increase of [TChl_a], and prochlorophytes became almost negligible at conditions with log ([TChl_a]) $>-0.3$. Since [TChl_a] roughly indicates the general spatial and seasonal distributions of phytoplankton (e.g. decreasing towards offshore and increasing from Summer to Winter-Spring), the variations of (FC) taxa_n with [TChl_a] imply the seasonal and spatial variations of phytoplankton community composition. These results are generally consistent with previous studies on the seasonal and spatial variations of phytoplankton community composition (Marshall, 1984a, 1984b; Marshall \& Cohn, 1987; Marshall et al., 2006) and cell count data used in this study.

The match-up comparisons showed that CHEMTAX-derived concentrations of phytoplankton functional groups generally agreed with the cell counts (Fig. 6). The CHEMTAX-derived concentrations generally increased with increases in the cell counts. Their relationships for some major phytoplankton functional groups, such as diatoms, cryptophytes, dinoflagellates (Dino_A+Dino_B), and cyanobateria, could even be expressed into log-transformed linear regressions (Fig. 6).

\subsection{Satellite imagery of phytoplankton community composition}

Because current empirical methodology is unable to derive some important accessory pigments (e.g. Hex and But) from remotely sensed imagery (Pan et al., 2010), and CHEMTAX chemotaxonomic analysis is not able to separate phytoplankton groups with similar pigment composition, such as chlorophytes and prasinophytes (Mackey et al., 1996), we aggregated the remotely sensed

Table 5

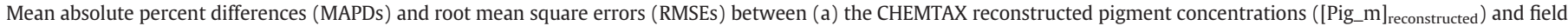

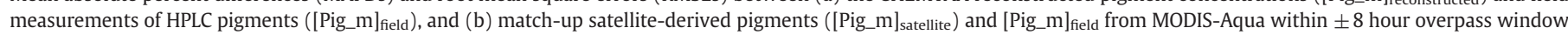
adapted from Pan et al. (2010). Median values of [Pig_m] $]_{\text {field }}$ were also shown for comparison.

\begin{tabular}{|c|c|c|c|c|c|c|c|c|c|c|c|}
\hline Parameters & TChl_a & TChl_b & TChl_c & Perid & Fuco & Dia & Allo & Zea & Lut & Neo & Viola \\
\hline Median & 1.987 & 0.149 & 0.395 & 0.070 & 0.575 & 0.148 & 0.074 & 0.043 & 0.006 & 0.019 & 0.019 \\
\hline \multicolumn{12}{|c|}{ (a) $[\text { Pig_m }]_{\text {reconstructed }} v s$. [Pig_m $]_{\text {field }}$} \\
\hline MAPD (\%) & 0 & 6.4 & 8.9 & 16.9 & 3.9 & 6.4 & 19.8 & 17.4 & 121.1 & 20.3 & 32.3 \\
\hline RMSE & 0 & 0.012 & 0.040 & 0.016 & 0.050 & 0.017 & 0.017 & 0.013 & 0.014 & 0.009 & 0.013 \\
\hline \multicolumn{12}{|c|}{ (b) $[\text { Pig_m } m]_{\text {satellite }} v s$. $[\text { Pig_m }]_{\text {field }}$} \\
\hline MAPD (\%) & 33.7 & 45.7 & 35.4 & 44.3 & 40.9 & 34 & 42.1 & 48.5 & 37.3 & 45.2 & 43.2 \\
\hline RMSE & 0.229 & 0.329 & 0.279 & 0.257 & 0.318 & 0.368 & 0.417 & 0.262 & 0.319 & 0.307 & 0.239 \\
\hline
\end{tabular}




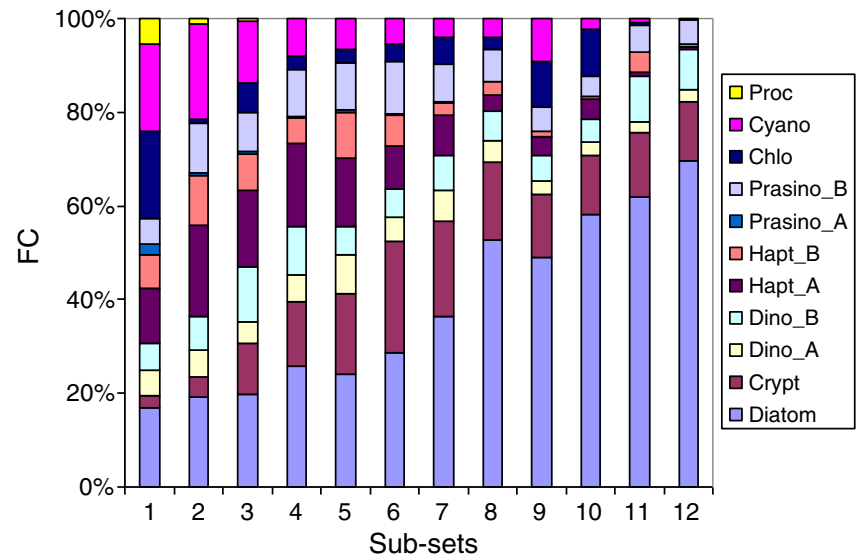

Fig. 5. Percentage fraction of TChl_a for each phytoplankton taxonomic group, FC, derived from CHEMTAX analysis on 12 sub-sets (Table 4) of HPLC pigment data. The nominal $\log \left(\left[\right.\right.$ TChl $\_$] $($median \pm 0.1$)$ for each sub-set was: sub-set $1(-0.8), 2$ $(-0.6), 3$ (-0.4), 4 (-0.2), 5 (0.0), $6(0.2), 7(0.4), 8(0.6), 9(0.8), 10(1.0), 11$ (1.2), and $12(1.4)$.

distributions of phytoplankton groups into six categories: diatoms (Diatom), cryptophytes (Crypt), dinoflagellates (Dino; sum of Dino_A and Dino_B), haptophytes (Hapt; sum of Hapt_A and Hapt_B), prasinophytes plus chlorophytes (Prasino\&Chlo), and picophytoplankton (Pico; sum of cyanobacteria and prochlorophytes). Figs. 7 and 8 showed examples of the spatial and seasonal distributions phytoplankton functional groups from 2006. Similar distributions could also be found on similar seasonal periods for the same or different years (data not shown). Except for pico-phytoplankton, the absolute concentrations of phytoplankton decreased from Winter-Spring to Summer and from the inner to outer shelf (Fig. 7).

This study region is known to be strongly impacted by the outflow plumes and along-shore flows (e.g. from Chesapeake and Delaware Bays in the SMAB, from Hudson-Raritan River-Estuary in the NYB, and from the Scotian Shelf in the GoM) (Acker et al., 2005; Harding et al., 2005; Marshall, 1984a; Marshall \& Cohn, 1987; O'Reilly \& Zetlin, 1998; Pan \& Zimmerman, 2010; Pan et al., 2008, 2010). High nutrient loading from terrestrial inputs has been related to enhanced phytoplankton biomass in coastal systems (Acker et al., 2005; Filippino et al., 2009; Harding et al., 2005; Smith, 2006). For example, the monthly mean freshwater discharge rates out of the Chesapeake Bay mouth were 2983, 1463, 729, and $4189 \mathrm{~m}^{3} \mathrm{~s}^{-1}$ for February, May, August, and November 2006, respectively (G. Fisher, U.S. Geological Survey, personal communication, 2007). This presumably led to transport of phytoplankton groups characteristic of the Chesapeake Bay estuary and plume region to offshore areas during November, February, and even May when freshwater discharge was significantly higher than during August (Fig. 7), consistent with other work (Filippino et al., 2009). In addition, diatom concentrations decreased by a factor of $>15$ from the inner shelf to the middle shelf in the SMAB during August, and by a factor of 4 to 9 during November, February, and May 2006 (Fig. 7). In contrast, variations in phytoplankton biomass from middle shelf to outer shelf were found inversely related to discharge rates, e.g. diatom concentrations decreased from the middle shelf to outer shelf in the SMAB by a factor of 2 to 4 during February, May, and November but 1 to 2 during August (Fig. 7). $C_{\text {taxa_n }}$ was also found high around the Nantucket Shoals and the Georges Bank, in which the geologically shallow water conditions favor the upwelling of nutrient-rich deep waters, especially during the Winter-Spring (Fig. 7). Diatoms and cryptophytes became almost negligible in offshore and basin waters, especially during Summer, in which Prasino\&Chlo (probably within the pico-size fraction identified as the dominant pico-eukaryotes; Marshall, 1984a) and pico-phytoplankton became more dominant (Fig. 7). Picophytoplankton (here primarily represented as cyanobacteria in the inner and middle shelf waters and prochlorophytes in the outer shelf and the basin waters) were more abundant during Summer and in the southern region than during Winter-Spring and in the northern region (Fig. 7), consistent with earlier observations showing a positive relationship between cyanobacteria and water temperature (Jeffrey et al., 1997; Reynolds \& Walsby, 1975) and the dominant status of prochlorophytes in the oligotrophic waters, especially in warm waters (Campbell \& Vaulot, 1993).

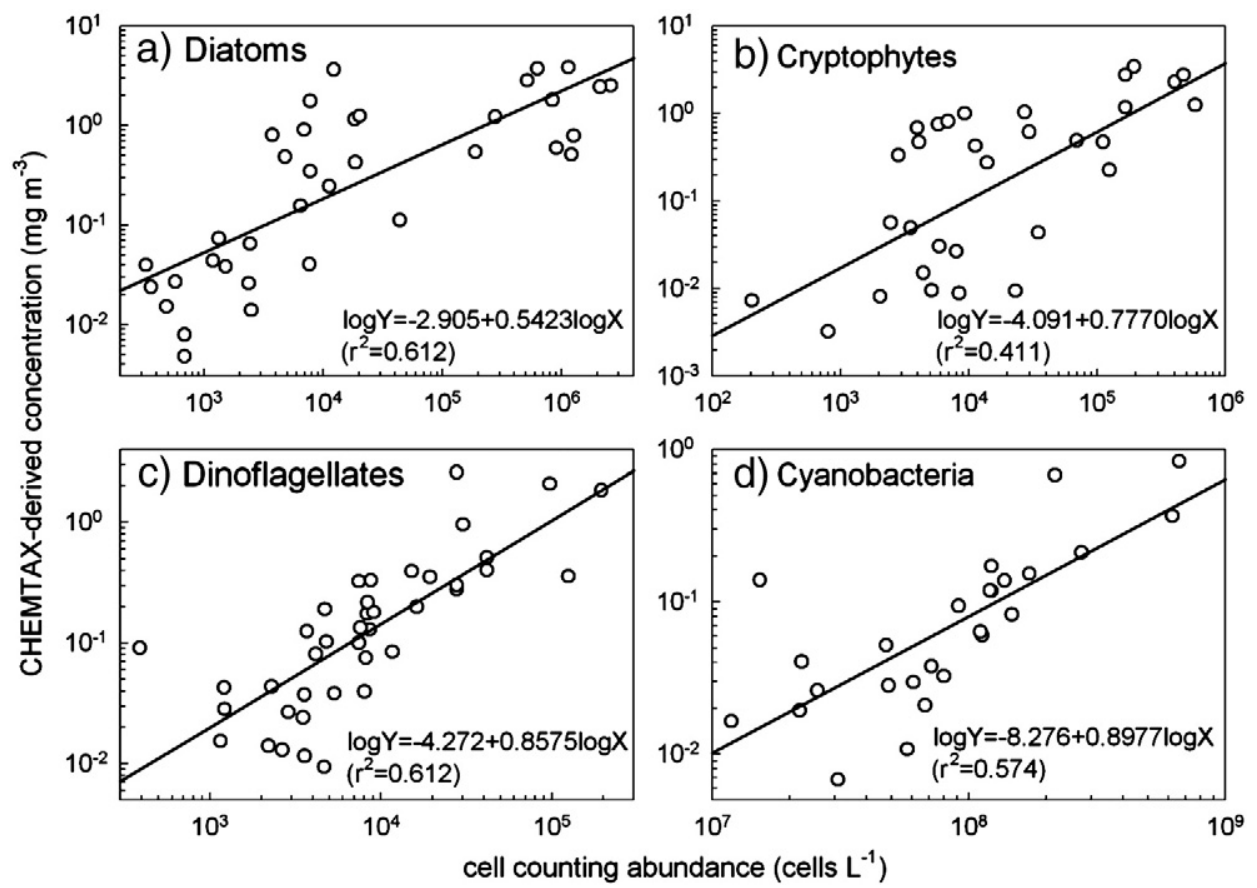

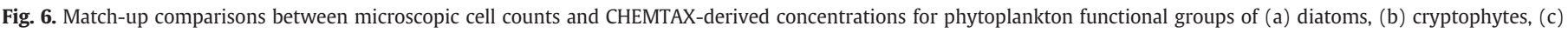

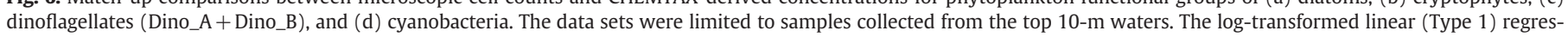
sions (solid lines and the equations) are also shown. 


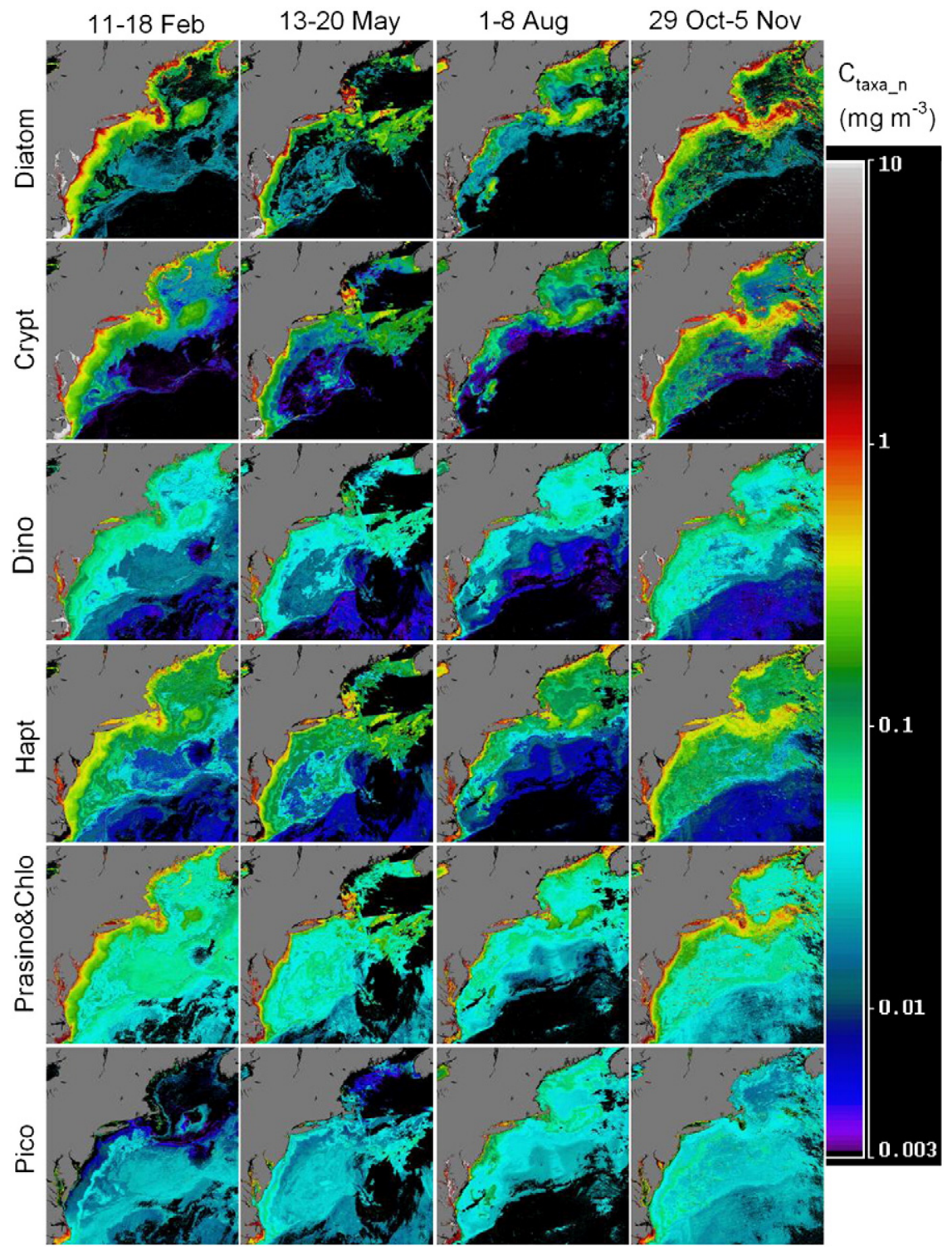

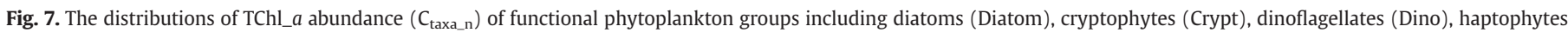

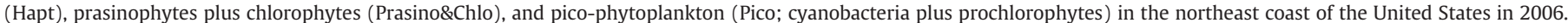
Each image represents an 8-day mean derived from MODIS-Aqua.

The distributions of (FC) $)_{\text {taxa_n }}$ showed that diatoms were the most abundant phytoplankton group on the inner shelf, accounting for 40$55 \%$ of [TChl_a] during Winter-Spring and about 30-45\% during Summer (Fig. 8). The abundance and proportion of diatoms decreased offshore as the impact from estuarine discharge and alongshore flows decreased (Fig. 8). For example, (FC) Diatom were relatively lower, from $40 \%$ on the inner shelf to $15 \%$ on the outer shelf, during August 2006 when discharge rates were lower $\left(729 \mathrm{~m}^{3} \mathrm{~s}^{-1}\right)$, and increased with increases in discharge rates, e.g. about $45-50 \%$ and $20-25 \%$ during May $\left(1463 \mathrm{~m}^{3} \mathrm{~s}^{-1}\right)$, February $\left(2983 \mathrm{~m}^{3} \mathrm{~s}^{-1}\right)$, and November $2006\left(4189 \mathrm{~m}^{3} \mathrm{~s}^{-1}\right)$ for the inner and outer shelf, respectively (Fig. 8). Cryptophytes typically showed similar trends to diatoms, but somewhat higher proportions were found in the inner and middle shelf other than the plume regions since in the plume regions cryptophyte blooms are obscured by diatom blooms, which was consistent with the results from HPLC and CHEMTAX analyses (Fig. 5). Such seasonal and spatial patterns of absolute and relative abundances of diatoms and cryptophytes agreed generally with phytoplankton cell abundance data from previous works, especially in offshore areas (>35 km from shore) (Table 6). Dinoflagellate proportions of the total phytoplankton community were somewhat higher on the inner shelf during Summer than during Winter-Spring and generally increased offshore (Fig. 8). Higher proportions of haptophytes were typically observed during late Spring to Summer (May and August) on the middle and outer shelf (Fig. 8). Also the seasonal and spatial patterns in the abundance of dinoflagellates and haptophytes relative to the total phytoplankton population agreed reasonably well with previous studies on phytoplankton abundance in the region (Table 6). Higher proportions of Prasino\&Chlo were found offshore while diatoms were dominant on the inner shelf (Fig. 8). Picophytoplankton proportions were typically very low at high [TChl_a] waters (Pan et al., 2010), especially on the inner and middle shelf during Winter-Spring, but increased during Summer and towards offshore (Fig. 8). Although our approaches were developed primarily for the shelf waters, the observations of very high proportions of picophytoplankton during Summer (Fig. 8), as well as their absolute concentrations (Fig. 7), might indicate cyanobacterial blooms in the middle and low regions of the Chesapeake Bay, in which cyanobacterial blooms are common during Summer (ECOHAB, 1994). These results were generally consistent with cell count data (Table 6) and previous 


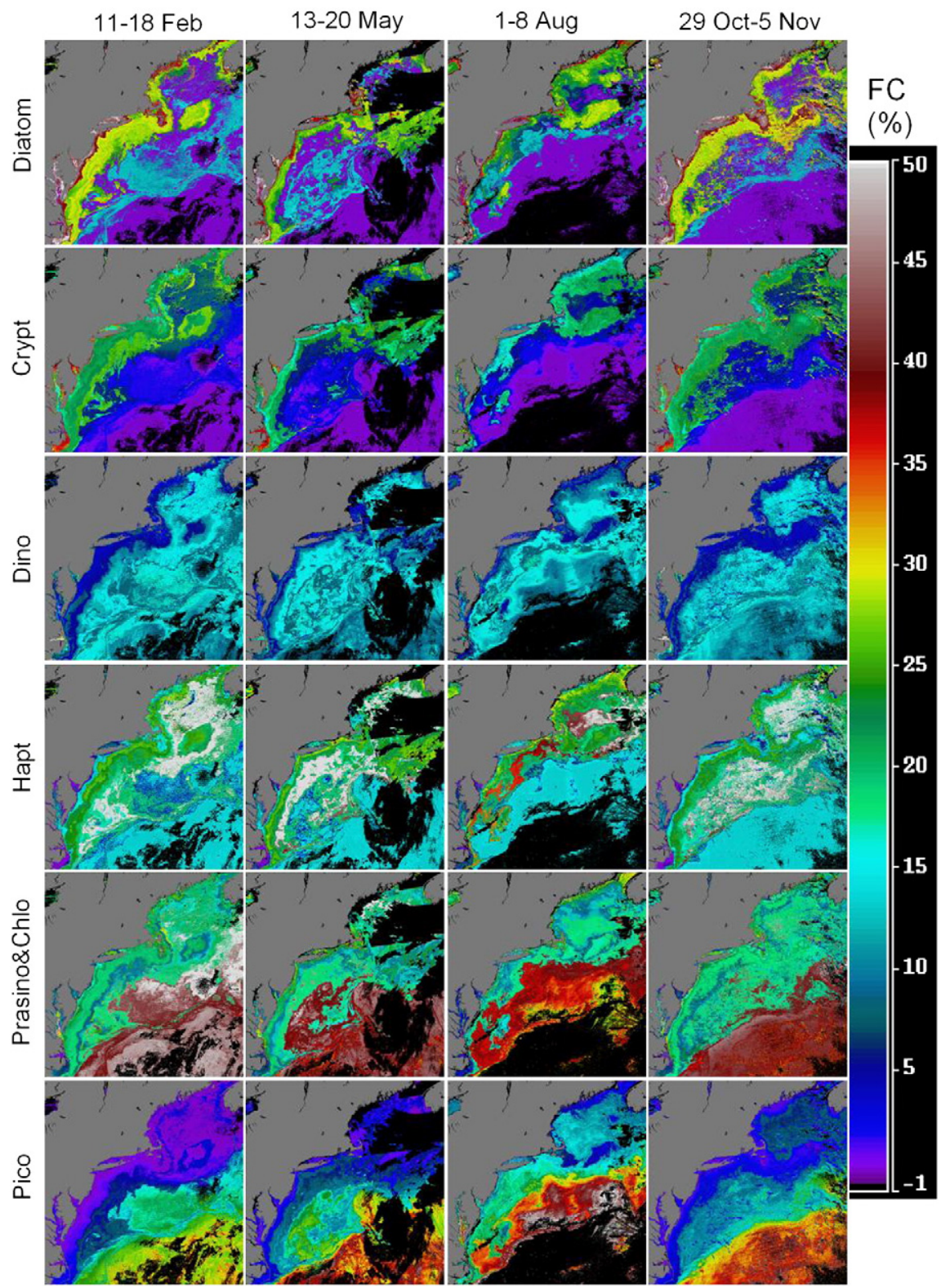

Fig. 8. The distributions of the percentage fraction of TChl_a for each phytoplankton group $\left[(\mathrm{FC})_{\text {taxa_n }}\right]$. See Fig. 7 for detailed description.

observations (Marshall, 1984a, 1984b; Marshall \& Cohn, 1987; Marshall et al., 2006).

\subsection{Time-series analysis of phytoplankton community composition from satellite observations}

The seasonal and spatial patterns in phytoplankton functional group distributions were further confirmed using time-series analysis done at two selected locations $\left(75.88^{\circ} \mathrm{W}, 36.96^{\circ} \mathrm{N}\right.$, and $74.83^{\circ} \mathrm{W}, 36.40^{\circ} \mathrm{N}$; Fig. 1) on the inner shelf and outer shelf areas of the Chesapeake Bay estuary in the SMAB. Diatoms were most abundant in the inner shelf, in which their abundances were about 2- to 5 -fold of cryptophytes, haptophytes, and Prasino\&Chlo, and 6- to 30-fold of dinoflagellates and pico-phytoplankton (Fig. 9). The dominant status of diatoms became less apparent in the outer shelf, in which diatom abundances were only 0.3 - to 2-fold of cryptophytes, haptophytes, and Prasino\&Chlo, and 0.5 - to 3-fold of dinoflagellates and pico-phytoplankton (Fig. 9). Except for pico-phytoplankton and haptophytes, phytoplankton abundances were generally higher during Winter-Spring than during Summer, with the ratios of 1.5 to 2.8 in the inner shelf and 1.4 to 9.4 in the outer shelf, respectively (Fig. 9). The seasonal variations of haptophytes were less significant in the inner shelf, but significant in the outer shelf in which their abundances during Winter-Spring were about 2.7-fold of those during Summer (Fig. 9). In contrast, picophytoplankton were more abundant (about 3-fold) during Summer than during Winter-Spring in the inner shelf, but their seasonal variations disappeared dramatically in the outer shelf (Fig. 9).

The relative abundances (FC) showed that diatoms accounted for about $37 \%$ of [TChl_a] in the inner shelf, following by $16 \%$ to $19 \%$ from cryptophytes, haptophytes, or Prasino\&Chlo, and $2 \%$ to $5 \%$ from dinoflagellates or pico-phytoplankton (Fig. 10). In the outer shelf, haptophytes and Prasino\&Chlo together accounted for about $50 \%$ of [TChl_a], while other phytoplankton accounted for the remaining $50 \%$ in relatively equal proportions (Fig. 10). The relative abundances in the inner shelf decreased from Winter-Spring to Summer for diatoms (42\% versus 32\%) and cryptophytes (20\% versus $16 \%$ ), and were relatively constant for dinoflagellates $(\sim 5 \%)$ and Prasino\& Chlo $(\sim 19 \%)$ but increased for haptophytes $(12 \%$ versus $20 \%)$ and pico-phytoplankton (1\% versus $7 \%$ ) (Fig. 10). Similarly, the relative abundances in the outer shelf decreased from Winter-Spring to 
Table 6

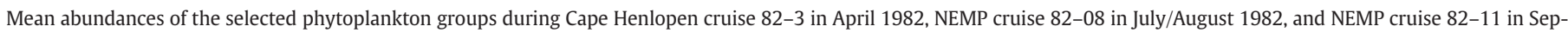

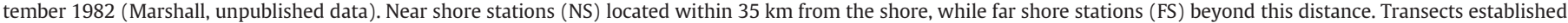

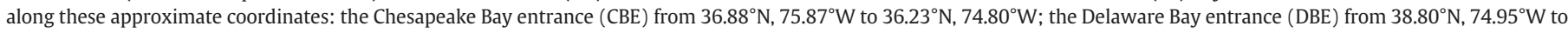
$38.13^{\circ} \mathrm{N}, 73.87^{\circ} \mathrm{W}$, and the New York Bight (NYB) from $40.45^{\circ} \mathrm{N}, 73.88^{\circ} \mathrm{W}$ to $39.65^{\circ} \mathrm{N}, 72.70^{\circ} \mathrm{W}$.

\begin{tabular}{|c|c|c|c|c|c|c|c|c|c|}
\hline \multirow[t]{2}{*}{ Regions } & \multirow[t]{2}{*}{ Seasons } & \multicolumn{2}{|c|}{ Diatoms } & \multicolumn{2}{|c|}{ Cryptophytes } & \multicolumn{2}{|c|}{ Dinoflagellates } & \multicolumn{2}{|c|}{ Coccolithophores } \\
\hline & & NS & FS & NS & FS & NS & FS & NS & FS \\
\hline \multicolumn{10}{|c|}{ Phytoplankton abundance $\left(10^{5}\right.$ cells $\left.L^{-1}\right)$} \\
\hline \multirow[t]{4}{*}{ CBE } & Apr & 6.088 & 1.236 & 1.336 & 0.923 & 0.283 & 0.416 & 0.296 & 0.609 \\
\hline & Jul/Aug & 5.816 & 4.187 & 0.116 & 0.065 & 0.855 & 0.187 & 0.217 & 3.713 \\
\hline & Sep & 0.971 & 0.504 & 0.519 & 0.365 & 0.494 & 0.513 & 0.164 & 0.146 \\
\hline & Mean & 4.292 & 1.976 & 0.657 & 0.451 & 0.544 & 0.372 & 0.226 & 1.490 \\
\hline \multirow[t]{4}{*}{ DBE } & Apr & 2.033 & 3.721 & 1.312 & 1.609 & 0.077 & 0.323 & 0.170 & 1.606 \\
\hline & Jul/Aug & 8.290 & 3.645 & 0.924 & 0.609 & 2.525 & 0.975 & 0.103 & 0.094 \\
\hline & Sep & 4.433 & 1.119 & 1.923 & 0.553 & 1.904 & 5.082 & 2.097 & 0.268 \\
\hline & Mean & 4.919 & 2.828 & 1.387 & 0.924 & 1.502 & 2.127 & 0.790 & 0.656 \\
\hline \multirow[t]{4}{*}{ NYB } & Apr & 10.135 & 2.344 & 1.678 & 0.480 & 0.470 & 0.310 & 0.641 & 0.160 \\
\hline & Jul/Aug & 1.192 & 1.109 & 0.143 & 0.401 & 1.341 & 0.620 & 0.044 & 0.086 \\
\hline & Sep & 4.459 & 0.123 & 0.966 & 0.450 & 0.714 & 0.406 & 0.402 & 1.109 \\
\hline & Mean & 5.262 & 1.192 & 0.929 & 0.444 & 0.842 & 0.446 & 0.362 & 0.451 \\
\hline \multicolumn{10}{|c|}{ Relatively abundance normalized to diatom cell counts } \\
\hline \multirow[t]{4}{*}{ CBE } & Apr & 1 & 1 & 0.219 & 0.747 & 0.046 & 0.337 & 0.049 & 0.493 \\
\hline & Jul/Aug & 1 & 1 & 0.020 & 0.016 & 0.147 & 0.045 & 0.037 & 0.887 \\
\hline & Sep & 1 & 1 & 0.535 & 0.724 & 0.509 & 1.018 & 0.169 & 0.290 \\
\hline & Mean & 1 & 1 & 0.153 & 0.228 & 0.127 & 0.188 & 0.053 & 0.754 \\
\hline \multirow[t]{4}{*}{ DBE } & Apr & 1 & 1 & 0.645 & 0.432 & 0.038 & 0.087 & 0.084 & 0.432 \\
\hline & Jul/Aug & 1 & 1 & 0.111 & 0.167 & 0.305 & 0.267 & 0.012 & 0.026 \\
\hline & Sep & 1 & 1 & 0.434 & 0.494 & 0.430 & 4.542 & 0.473 & 0.239 \\
\hline & Mean & 1 & 1 & 0.282 & 0.327 & 0.305 & 0.752 & 0.161 & 0.232 \\
\hline \multirow[t]{4}{*}{ NYB } & Apr & 1 & 1 & 0.166 & 0.205 & 0.046 & 0.132 & 0.063 & 0.068 \\
\hline & Jul/Aug & 1 & 1 & 0.120 & 0.362 & 1.125 & 0.559 & 0.037 & 0.078 \\
\hline & Sep & 1 & 1 & 0.217 & 3.659 & 0.160 & 3.301 & 0.090 & 9.016 \\
\hline & Mean & 1 & 1 & 0.177 & 0.372 & 0.160 & 0.374 & 0.069 & 0.378 \\
\hline
\end{tabular}

Summer for diatoms (16\% versus $8 \%$ ), cryptophytes (13\% versus $3 \%$ ) and haptophytes (29\% versus $23 \%$ ), and were relatively constant for dinoflagellates $(\sim 12 \%)$ but increased for Prasino\&Chlo (20\% versus $33 \%$ ) and pico-phytoplankton (9\% versus $21 \%$ ) (Fig. 10). Similar seasonal and spatial patterns were also found in the Delaware Bay estuary and New York Bight, but became less significant in the semienclosed Gulf of Maine (data not shown).

The spatial distributions and the time-series patterns of phytoplankton composition derived from CHEMTAX method agreed reasonably with cell count observations. Phytoplankton composition over the U.S. northeastern continental shelf including studies involving NOAA National Marine Fisheries Service (NMFS) based cruises from 1969 through 1985 showed over 600 algal taxa recorded for this region (Marshall \& Ranasinghe, 1989). The major components included diatoms, a diverse group of phytoflagellates, plus pico-phytoplankton $(<3 \mu \mathrm{m})$ composed predominantly of cyanobacteria and chlorophytes (Marshall, 1984a, 1984b). A previous study showed that a total phytoplankton abundance near shore (up to $35 \mathrm{~km}$ seaward) ranging from $10^{5}$ to $10^{7}$ cells $\mathrm{L}^{-1}$, that generally decreased to $10^{4}$ to $10^{5}$ cells $\mathrm{L}^{-1}$ across the mid-shelf and increased again along the outer shelf margin (Marshall, 1984b). This is generally consistent with the phytoplankton composition derived here using satellite data, e.g. an increase of phytoplankton concentrations by a factor of 1 to 2 orders of magnitude from the outer to inner shelf (Figs. 7 and 9). Within the MAB estuarine plumes (Hudson, Delaware, and Chesapeake Bays), estuarine outflow increased phytoplankton concentrations seasonally and influenced species composition and diversity (Marshall, 1984a; Marshall \& Cohn, 1987; this study, Figs. 7-10).

Phytoplankton abundance from previous studies with station locations comparable to the present study were also presented in Table 6. These samples were taken aboard from one U.S. Coast Guard and two NOAA's NorthEast Monitoring Program (NEMP) cruises $(82-03,82-08,82-11)$ and subsequently analyzed for phytoplankton composition and abundance (Marshall, unpublished data).
A minimum of 3 near-shore and 3 off-shore stations yielded mean phytoplankton concentrations for these regions during April, July/ August, and September 1982. These values illustrated the monthly abundance varied from $10^{4}$ to $10^{6}$ cells $\mathrm{L}^{-1}$ for diatoms, and $10^{3}$ to $10^{5}$ cells $\mathrm{L}^{-1}$ for cryptophytes, coccolithophores, and dinoflagellates. Diatoms were consistently the dominant flora within near shore waters and along transects directed across the shelf for all seasons. Phytoflagellates were also dominant constituents within these waters. At lower concentrations and with seasonally varying abundance were cyanobacteria, chlorophytes, and pico-phytoplankton. The 1982 data, as well as the cell counts conducted in our cruises from 2006 to 2009, are consistent with our seasonal and spatial phytoplankton function group derivations from satellite observations (Figs. 7-10).

Time-series analyses of phytoplankton assemblages from this study indicate generally negative correlations between concentrations of micro- and nano-phytoplankton groups (diatoms, dinoflagellates, cryptophytes, haptophytes, and Prasino\&Chlo) and sea surface temperature (SST) in the outer shelf areas (Table 7). The negative correlations between SST and phytoplankton abundance or TChl_a were generally more significant in the south regions (e.g. the Chesapeake Bay estuary and the Delaware Bay estuary) than in the north region (e.g. the New York Bight and the Gulf of Maine) (Table 7), consistent with the suggestion by Richardson and Schoeman (2004) for the northeast Atlantic. Such results imply that the nutrient availability is generally the major limiting factor for phytoplankton growth in the offshore region. With the deepening of the surface mixed layer by surface cooling in Winter, as well as stronger winds, more nutrients are transported to the surface mixed layer to stimulate phytoplankton growth. In the other hand, the shallowness of the surface mixed layer due to strong vertical stratification in Summer creates a nutrientdepleted surface mixed layer that may reduce micro- and/or nanophytoplankton growth within the surface mixed layer. Because pico-phytoplankton generally compete successfully over micro- and 


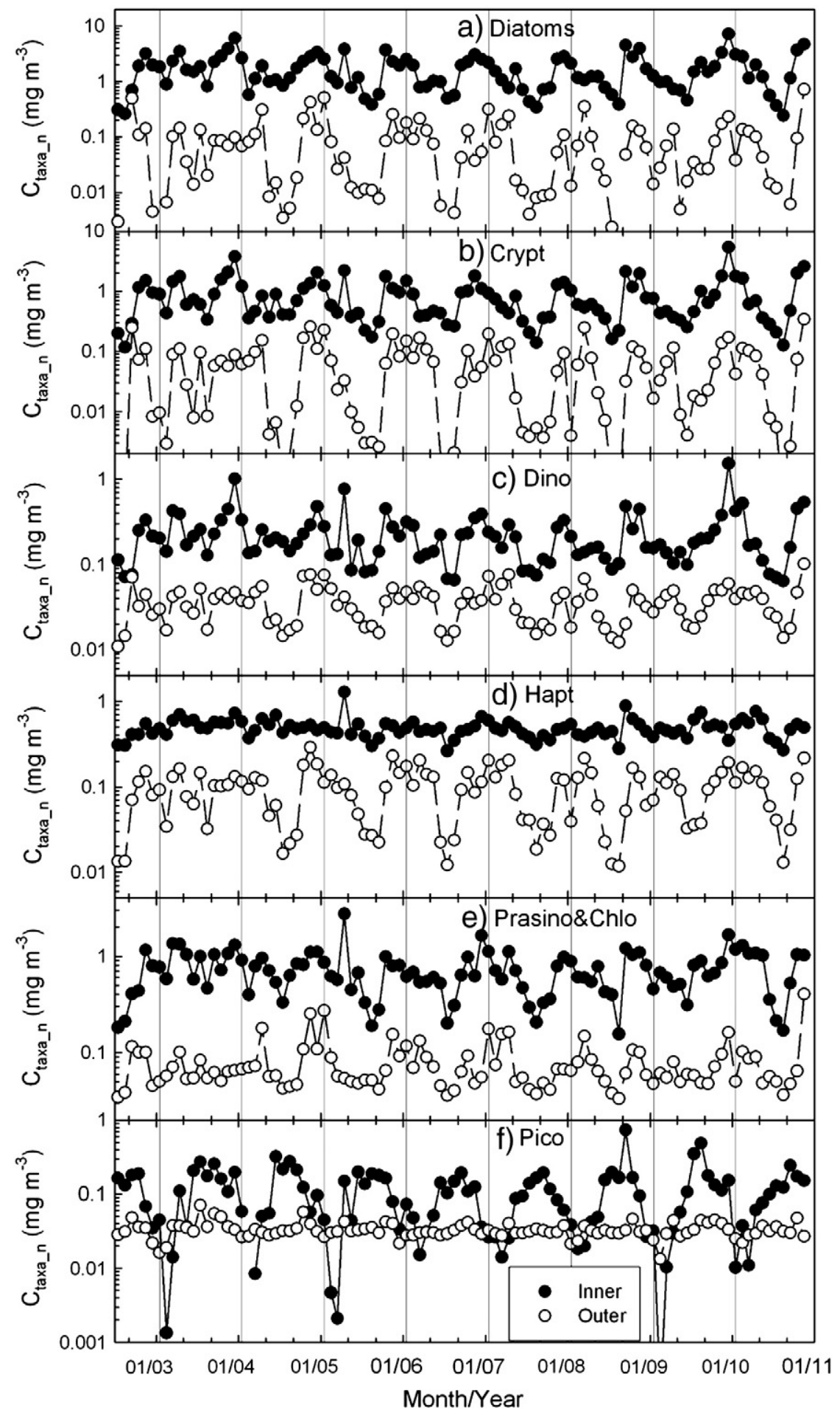

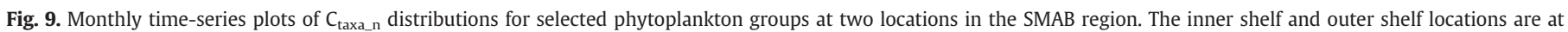
$75.88^{\circ} \mathrm{W}, 36.96^{\circ} \mathrm{N}$ and $74.83^{\circ} \mathrm{W}, 36.40^{\circ} \mathrm{N}$, respectively. The selected phytoplankton groups include (a) Diatom, (b) Crypt, (c) Dino, (d) Hapt, (e) Prasino\&Chlo, and (f) Pico.

nano-phytoplankton in nutrient-depleted water conditions, especially in Summer, the correlations between SST and pico-phytoplankton abundances were significantly positive (Table 7). The correlations between SST and phytoplankton abundances in the inner shelf were more complex (Table 7), depending on the major limiting factor (nutrients, light penetration, light intensity, or photoperiod) for phytoplankton growth. For example, Harding et al. (1986) indicated that the major limiting factor was nutrient availability in the Chesapeake Bay estuary (CBE) but light penetration in the Delaware Bay estuary
(DBE). Consequently, the increasing supply of nutrients in Winter caused more apparent stimulation effect for phytoplankton growth in the $\mathrm{CBE}$ than in the DBE, as shown by more significant negative correlations between SST and phytoplankton abundances in the CBE than in the DBE (Table 7). In the GoM and even the NYB, light availability (light intensity and photoperiod) is the major limiting factor for phytoplankton growth during Winter. Phytoplankton blooms typically occur in late Spring or early Summer as observed from our serial cruises in the GoM. 

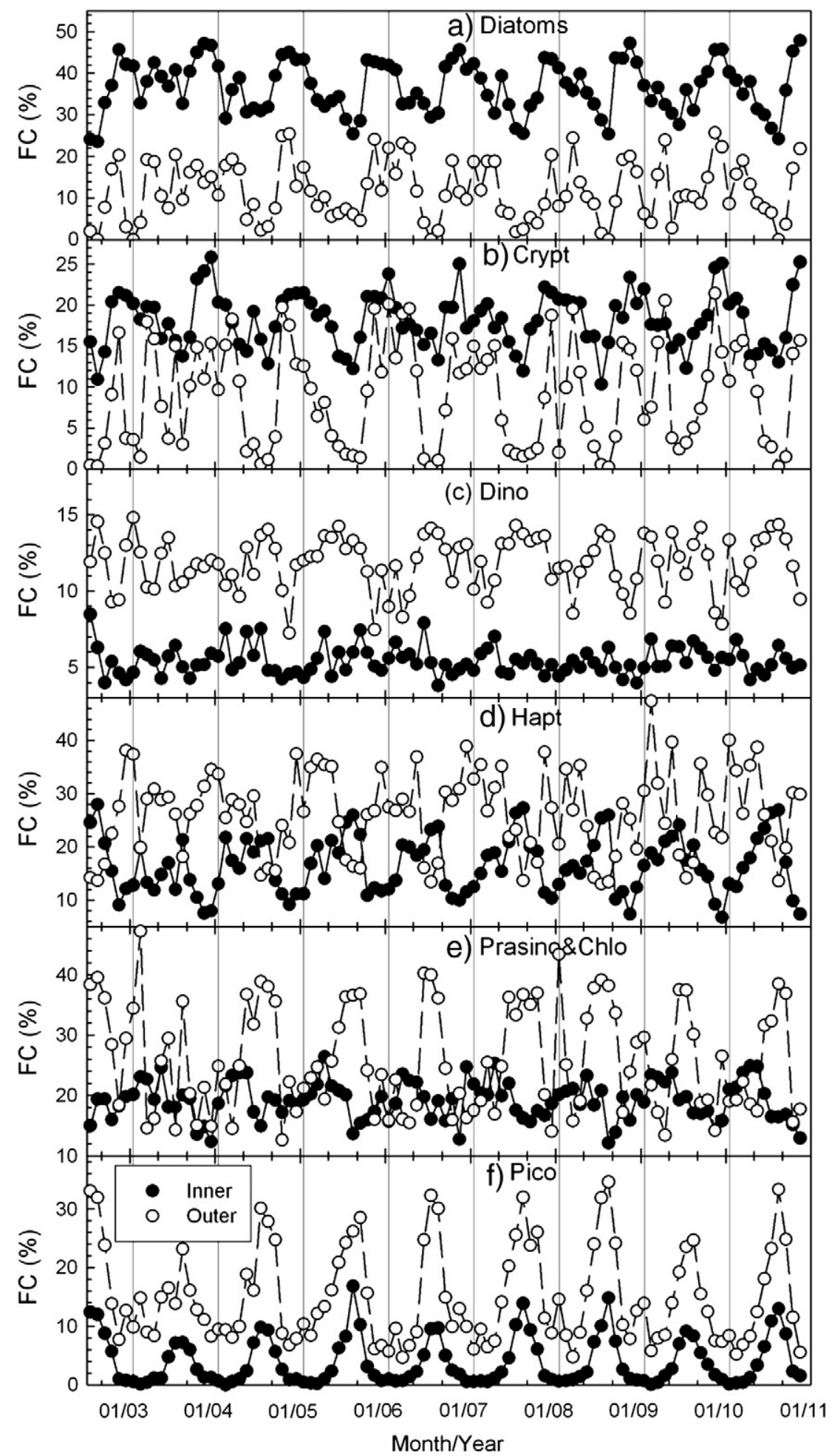

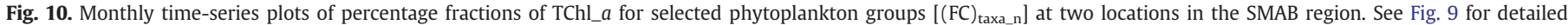
description.

\section{Application and discussion}

\subsection{CHEMTAX taxonomic groups and limitations to our approach}

Phytoplankton functional groups using CHEMTAX analysis should be selected with caution. Some uncommon species, such as euglenophytes, should be excluded from CHEMTAX analysis for this region, or the calculations would result in unreasonably high concentrations of euglenophytes (sometimes even higher than diatoms) (unpublished data). Other uncommon species, such as Prasino_A and prochlorophytes, could be included to CHEMTAX analysis, partly due to their unique pigments (e.g. prasinoxanthin and DV_Chl_a). Cell count data are essential to evaluate the quality of the CHEMTAX outputs.

The categories of CHEMTAX taxonomic groups are based on pigment ratios within phytoplankton from particular taxonomic groups. Many phytoplankton functional groups have similar pigment ratios, 
Table 7

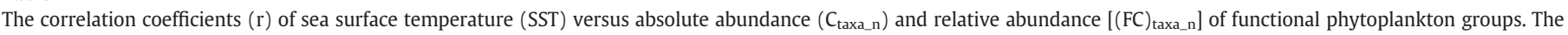

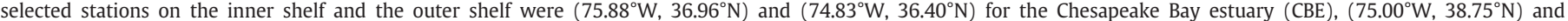

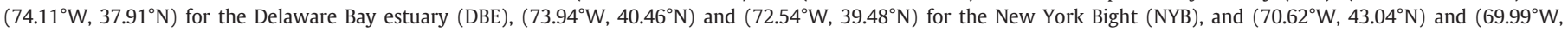

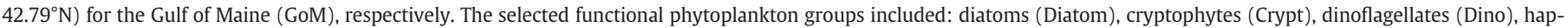
tophytes (Hapt), prasinophytes plus chlorophytes (Prasino\&Chlo), and picophytoplankton (Pico; cyanobacteria plus prochlorophytes).

\begin{tabular}{|c|c|c|c|c|c|c|c|c|}
\hline \multirow[t]{2}{*}{ Parameters } & \multicolumn{2}{|l|}{ CBE } & \multicolumn{2}{|l|}{$\mathrm{DBE}$} & \multicolumn{2}{|l|}{ NYB } & \multicolumn{2}{|l|}{ GoM } \\
\hline & Inner & Outer & Inner & Outer & Inner & Outer & Inner & Outer \\
\hline [TChl_a] & -0.16 & -0.22 & 0.03 & -0.31 & 0.07 & -0.12 & -0.01 & -0.08 \\
\hline $\mathrm{C}_{\text {Diatom }}$ & -0.16 & -0.14 & -0.03 & -0.23 & -0.02 & -0.08 & 0.08 & -0.08 \\
\hline $\mathrm{C}_{\text {Crypt }}$ & -0.14 & -0.22 & -0.02 & -0.34 & 0.04 & -0.14 & 0.05 & -0.07 \\
\hline $\mathrm{C}_{\text {Dino }}$ & -0.12 & -0.26 & -0.04 & -0.40 & 0.03 & -0.28 & 0.03 & -0.09 \\
\hline $\mathrm{C}_{\text {Hapt }}$ & -0.09 & -0.47 & 0.07 & -0.46 & 0.15 & -0.35 & 0.02 & -0.10 \\
\hline $\mathrm{C}_{\text {Prasino\&Chlo }}$ & -0.21 & -0.19 & 0.04 & -0.20 & 0.08 & -0.09 & 0.01 & -0.09 \\
\hline $\mathrm{C}_{\text {Pico }}$ & 0.40 & 0.20 & 0.27 & 0.42 & 0.21 & 0.24 & 0.22 & 0.50 \\
\hline$(\mathrm{FC})_{\text {Diatom }}$ & -0.28 & -0.35 & -0.14 & -0.37 & -0.24 & -0.30 & 0.02 & 0.04 \\
\hline$(\mathrm{FC})_{\text {Crypt }}$ & -0.32 & -0.51 & -0.25 & -0.52 & -0.35 & -0.53 & -0.19 & -0.08 \\
\hline$(\mathrm{FC})_{\text {Dino }}$ & 0.02 & 0.25 & -0.05 & 0.21 & -0.10 & 0.21 & 0.03 & -0.02 \\
\hline$(\mathrm{FC})_{\mathrm{Hapt}}$ & 0.32 & -0.36 & 0.19 & -0.39 & 0.36 & -0.40 & -0.16 & -0.13 \\
\hline$(\mathrm{FC})_{\text {Prasino\&Chlo }}$ & -0.21 & 0.41 & 0.00 & 0.37 & 0.17 & 0.36 & 0.10 & -0.18 \\
\hline$(\mathrm{FC})_{\text {Pico }}$ & 0.80 & 0.77 & 0.77 & 0.82 & 0.69 & 0.85 & 0.45 & 0.81 \\
\hline
\end{tabular}

such as dinoflagellate species containing fucoxanthin-like pigments (Dino_A) versus diatoms, prasinophytes versus chlorophytes, and cyanobacteria versus prochlorophytes (Table 4). This can make it difficult to distinguish between these groups by using CHEMTAX (Mackey et al., 1996). As for CHEMTAX analysis, the ability to accurately determine phytoplankton community composition from ocean color remote sensing depends on the ability to quantify particular phytoplankton pigments (versus bulk TChl_a) using ocean color sensors, especially for species- or taxa-specific pigments. For example, the ability to derive 19'-butanoyloxyfucoxanthin (But) and 19'-hexanoyloxyfucoxanthin (Hex) from satellite data makes it easy to distinguish chrysophytes and haptophytes from other golden-brown algae and provides a useful tool for assessing the distributions of these important phytoplankton groups. Unfortunately, such remotely sensed approaches have not been developed at this time (Pan et al., 2010).

The satellite-derived phytoplankton community composition developed in this paper is dependent on two independent approaches (Fig. 3): (1) ocean color algorithms to derive pigment concentrations (Approach_1; Pan et al., 2010), and (2) CHEMTAX-derived pigment matrices for selected phytoplankton functional groups (Approach_2; Table 4). Compared with the uncertainty attributed to Approach_1, the uncertainty associated with Approach_2 is generally small. As shown in Table 5, the MAPDs and RMSEs between the derived or reconstructed pigments and field measurements from Approach_1 were generally much higher than those from Approach_2. Therefore, developing appropriate ocean color algorithms with reasonable agreement to field data becomes a bottleneck for studying phytoplankton community composition from space.

\subsection{Cell counts versus chemotaxonomy}

Comparisons of phytoplankton community composition made using microscopic cell counts versus CHEMTAX derivations should be conducted with caution. Microscopic analysis can also be biased. For example, it is difficult to count fragile cells. There is differential preservation of phytoplankton depending on the various preservatives used. Pico-phytoplankton are often underestimated by direct microscopic counts. Physiological variability of cells within particular groups can also bias chemotaxonomic results. For example, cellular pigment concentrations in phytoplankton vary not only between species and taxonomic groups but also with environmental factors such as light, nutrient availability, and physiological stage of cells (Lewitus et al., 2005; Mackey et al., 1996; Moisan \& Mitchell, 1999; Schlüter et al., 2000; Zapata et al., 2004). Cells adapted to a high light environment may have lower cellular concentrations of photosynthetic pigments but higher concentrations of photoprotective pigments (Lewitus et al., 2005; Schlüter et al., 2000). Therefore, the pigment matrices and results presented here should be validated before being applied to other regions, even though the derived matrices were based on sub-sets across a wide range of [TChl_a] (Table 4).

Without considering the significant variations of cellular pigment concentrations over seasons and regions, seasonal and spatial patterns of the CHEMTAX-derived concentrations of phytoplankton functional groups may significantly differ from abundances determined using cell counts. For example, CHEMTAX-derived concentrations of dinoflagellates from this study seemed somewhat higher during WinterSpring than during Summer, especially on the inner shelf (Figs. 7 and 9). The monthly means of $C_{\text {Dino }}$ from a location on the inner shelf of the SMAB $\left(-75.88^{\circ} \mathrm{W}, 36.96^{\circ} \mathrm{N}\right)$ were $0.283,0.114$, and $0.200 \mathrm{mg} \mathrm{m}^{-3}$ during April, July/August, and September, respectively (Fig. 9), indicating dinoflagellate concentrations during July/August were only half that observed during April and September. The historical observations based on cell counts (Marshall \& Cohn, 1987), however, showed generally higher abundance of dinoflagellates during Summer than during other seasons, e.g. 2.9- to 32.8-fold and 1.3- to 1.9-fold increase in dinoflagellate cell abundance during July/August as compared to those during April and September in the near-shore region (within $35 \mathrm{~km}$ from the shore) (Table 6). The cellular pigment concentrations of dinoflagellates during summer may be several folds lower than during other seasons, e.g. cellular [Perid] varied from 1.3 to $3.7 \mathrm{pg} \mathrm{cell}^{-1}$ during July/August to 7.8 to $62 \mathrm{pg}$ cell $^{-1}$ during November from our database. As also indicated in Fig. 6, the dinoflagellate cell abundances can vary over 10 -fold for a given CHEMTAX-derived concentration. Such significant variations of cellular pigments should be accounted for to exploit the significant seasonal and spatial variability of relationships between CHEMTAX-derived concentrations and cell counts.

The physiological stage of cells varies diurnally and seasonally and needs to be considered in using CHEMTAX derivations of phytoplankton community composition. For example, many dinoflagellates are mixotrophic and have notoriously flexible metabolisms (Bockstahler \& Coats, 1993; Jeffrey et al., 1997; Li et al., 1999). The degree to which these organisms are autotrophic versus heterotrophic at any one time may affect their pigment composition. It is reasonable to assume that: a) cellular pigment concentrations, especially for photosynthetic pigments, were lower in autotrophic dinoflagellates than in heterotrophic forms because pigments digested by the latter were kept in the cell (Li et al., 1999); and b) cellular pigment concentrations in the autotrophic forms were higher under low-light 
environment than under high-light environment (Lewitus et al., 2005; Schlüter et al., 2000); and c) higher proportion of heterotrophic forms occurred under low-light environment than under high-light environment (Bockstahler \& Coats, 1993; Li et al., 1999). Ignoring the spatial difference, such variations of cellular pigment concentrations and the degree of autotrophic versus heterotrophic forms may together account for the variations of cellular [Perid], which was higher in early morning and had a second-peak in later morning, and which was generally higher during Winter-Spring than Summer, e.g. 41.0, 40.8, 6.3, and 7.8 pg cell $^{-1}$ on local 9:03, 10:03, 11:20, and 13:35 during CBP_5 cruise on 28 November 2006; and 2.9, 1.2, and 1.4 pg cell ${ }^{-1}$ on local 9:24, 10:40, and 11:46 during CBP_8 cruise on 3 July 2007.

One of the advantages of the chemotaxonomic approach over the microscopic cell counting is that the former can be used to quantify some phytoplankton functional groups which are difficult to detect by the latter technique (Mackey et al., 1996). For example, coccolithophorids might be underestimated by microcopy using Lugol's preservation since the acidic Lugol solution may cause dissolution of coccoliths on the cell surface of coccolithophorids. Chlorophytes might also be underestimated from microscopy since some of them might be found in a significant proportion of the pico-phytoplankton (Marshall, 1984b; Vaulot et al., 2008). The CHEMTAX derivations showed that Prasino\&Chlo might be present at similar or even higher order of magnitude than diatoms, especially in the offshore regions (Figs. 5-8), but chlorophytes could not be detected microscopically from many of cell counting samples.

\subsection{Phytoplankton community composition, global changes, and social-economical impacts}

Understanding general changes in trends of phytoplankton community composition is crucial for understanding how climate change impacts aquatic biology. The response of phytoplankton composition and abundance to climate change will have profound effects on higher trophic levels through bottom-up processes, which in turn impacts phytoplankton community through top-down control mechanisms (Edwards \& Richardson, 2004; Hays et al., 2005; Montes-Hugo et al., 2009; Richardson \& Schoeman, 2004). Edwards and Richardson (2004) showed a strong response of marine pelagic communities to global change, and a mismatch in the response between phytoplankton versus that of higher trophic levels (e.g. zooplankton and economic fish), and this mismatch partly accounted for the decline of fish stocks. For example, over the past 45 years, high abundance of diatom and dinoflagellate now increase 33 and 27 days earlier in the year, respectively, in response to increasing water temperatures in the North Sea, while copepod abundance occurs only 11 days earlier over the same period (Hays et al., 2005). This has resulted in decreased energy flow to higher trophic levels. Montes-Hugo et al. (2009) reported that a decrease in TChl_ $a$ in the northern part of the western Antarctic Peninsula (WAP) accompanied by a decrease in the relative abundance of diatoms (and an opposite effect on the southern part of the WAP) associated with rapid regional warming, coincided with the changes in the distribution of krill and penguin populations. Global warming is also cited as a causative factor in the expansion poleward in the range of tropical and temperate phytoplankton species (e.g. Ceratium trichoceros), and resulting in shifts in local and regional phytoplankton communities (Hays et al., 2005). The ability of satellite remote sensing to provide information on phytoplankton community composition will improve our understanding of how climate change impacts marine ecosystems. Our results suggest that we can expect to see shifts in phytoplankton community composition as surface waters continue to warm. For example, Fig. 9 indicated that the annual mean concentrations of phytoplankton functional groups between 2003 and 2009 decreased over the years for all phytoplankton in both inner and outer stations ( $\mathrm{r}$ ranging from -0.2 to -0.7$)$ except pico-phytoplankton in the inner shelf station $(r=0.1)$.

\section{Conclusions}

This paper shows that ocean color remote sensing can be used to determine the phytoplankton community composition in the northeast coast of the United States, although exploiting such derivations should be, to some degree, applied with caution as compared to cell count abundance. Spatial and seasonal variations of satellite-derived phytoplankton taxonomic groups from this study generally agreed with the cell count observations and with previous work (e.g. Marshall, 1984a, 1984b; Marshall \& Cohn, 1987; Marshall \& Ranasinghe, 1989; Marshall et al., 2006). Cell abundances of some major phytoplankton functional groups (e.g. diatoms, cryptophytes, dinoflagellates, and cyanobacteria) can potentially be estimated from CHEMTAX-derived concentrations (Fig. 6). Diatoms were generally the most abundant phytoplankton species in this region, especially during winter-spring and on the inner shelf. Other phytoplankton groups tended to co-occur with diatoms (Marshall, 1984a, 1984b; Marshall \& Cohn, 1987; Marshall et al., 2006). High abundances of pico-phytoplankton were typically observed during summer when diatom abundance was lower. Although our study showed the distributions of $C_{\text {taxa_n }}$, the application of these satellite products to the study of phytoplankton community structure should be conducted with caution due to the significant variations in cellular pigment concentrations with environmental factors and physiological status. The method developed from this study, however, proved reasonable to determine the intended phytoplankton groups, especially if they were relatively abundant or had fairly unique pigment compositions.

\section{Acknowledgments}

This research was supported by an appointment to the NASA Postdoctoral Program at the Goddard Space Flight Center, administered by Oak Ridge Associated Universities through a contract with NASA. The work was supported by the NASA Ocean Biology and Biogeochemistry Program, Interdisciplinary Science, Biodiversity, New Investigator Program, Carbon Cycle Science and Earth Observing System programs. We thank M. Twardowski for planning the New York Bight cruises. We are grateful to T. Egerton and C. Burbage at Old Dominion University (ODU) and D. Borkman at University of Rhode Island for providing phytoplankton cell count data, and R. Zimmerman at ODU for his HPLC data submitted to the NASA SeaBASS database. We thank the captains and crews of R/V Cape Henlopen, Hugh R. Sharp, Gulf Challenger, Connecticut, and Fay Slover. We are grateful to L. Van Heukelem and C. Thomas at Horn Point Laboratory for analyzing HPLC pigments, and the Ocean Biology Processing Group (OBPG) at GSFC for satellite data processing and distribution.

\section{References}

Acker, J. G., Harding, L. W., Leptoukh, G., Zhu, T., \& Shen, S. (2005). Remotely-sensed chl a at the Chesapeake Bay mouth is correlated with annual freshwater flow to the Chesapeake Bay. Geophysical Research Letters, 32, L05061. doi:10.1029/2004GL021852.

Adolf, J. E., Yeager, C. L., Miller, W. D., Mallonee, M. E., \& Harding, L. W. (2006). Environmental forcing of phytoplankton floral composition, biomass, and primary productivity in Chesapeake Bay, USA. Estuarine Coastal and Shelf Science, 67, 108-122. doi: 10.1016/j.ecss.2005.11.030.

Aiken, J., Hardman-Mountford, N. J., Barlow, R., Fishwick, J., Hirata, T., \& Smyth, T. (2008). Functional links between bioenergetics and bio-optical traits of phytoplankton taxonomic groups: An overreaching hypothesis with application for ocean colour remote sensing. Journal of Plankton Research, 30, 165-181.

Aiken, J., Pradhan, Y., Barlow, R., Lavender, S., Poulton, A., Holligan, P., et al. (2009). Phytoplankton pigments and functional types in the Atlantic Ocean: A decadal assessment, 1995-2005. Deep-Sea Research II, 56, 899-917. doi:10.1016/j.dsr2.2008.09.017.

Alvain, S., Moulin, C., Dandonneau, Y., \& Loisel, H. (2008). Seasonal distribution and succession of dominant phytoplankton groups in the global ocean: A satellite view. Global Biogeochemical Cycles, 22, GB3001. doi:10.1029/2007GB003154.

Behrenfeld, M. J., Boss, E., Siegel, D. A., \& Shea, D. M. (2005). Carbon-based ocean productivity and phytoplankton physiology from space. Global Biogeochemical Cycles, 19, GB1006. doi:10.1029/2004GB002299.

Behrenfeld, M. J., \& Falkowski, P. G. (1997). Photosynthetic rates derived from satellitebased chlorophyll concentration. Limnology and Oceanography, 42(1), 1-20. 
Bockstahler, K. R., \& Coats, D. W. (1993). Grazing of the mixotrophic dinoflagellate Gymnodinium sanguineum on ciliate populations of Chesapeake Bay. Marine Biology, $116,477-487$

Brewin, R. J. W., Hardman-Mountford, N. J., Lavender, S. J., Raitsos, D. E., Hirata, T., Uitz, J., et al. (2011). An intercomparison of bio-optical techniques for detecting dominant phytoplankton size class from satellite remote sensing. Remote Sensing of Environment, $115,325-339$

Brewin, R. J. W., Sathyendranath, S., Hirata, T., Lavender, S., Baraciela, R. M. \& HardmanMountford, N. (2010). A three-component model of phytoplankton size class for the Atlantic ocean. Ecological Modeling, 221(11), 1472-1483.

Campbell, L., \& Vaulot, D. (1993). Photosynthetic picoplankton community structure in the subtropical North Pacific Ocean near Hawaii (station ALOHA). Deep Sea Research I, 40(10), 2043-2060.

Ecology and Oceanography of Harmful Algal Blooms (ECOHAB) workshop report (1994). Snow Mountain Ranch Conference Center, Colorado, 23-28 August 1994. http://www.whoi.edu/science/B/redtide/nationplan/ECOHAB/ECOHABhtml.htm Accessed 23 July 2009.

Edwards, M., \& Richardson, A. J. (2004). Impact of climate change on marine pelagic phenology and trophic mismatch. Nature, 430, 881-884

Filippino, K. C., Bernhardt, P. W., \& Mulholland, M. R. (2009). Chesapeake Bay plume morphology and the effects on nutrient dynamics and primary productivity in the coastal zone. Estuaries and Coasts, 32, 410-424.

Gibb, S. W., Cummings, D. G., Irigoien, X., Barlow, R. G., Fauzi, R., \& Mantoura, C. (2001). Phytoplankton pigment chemotaxonomy of the northeastern Atlantic. Deep Sed Research II, 48, 795-823.

Harding, L. W., Magnuson, A., \& Mallonee, M. E. (2005). SeaWiFS retrievals of chlorophyll in Chesapeake Bay and the mid-Atlantic bight. Estuarine, Coastal and Shelf Science, 62, 75-94.

Harding, L. W., Messon, B., \& Fisher, T. (1986). Phytoplankton production in two east coast estuaries: Photosynthesis-light functions and patterns of carbon assimilation in Chesapeake and Delaware Bays. Estuarine, Coastal and Shelf Science, 23, 773-806.

Hays, G. C., Richardson, A. J., \& Robinson, C. (2005). Climate change and marine plankton. Trends in Ecology \& Evolution, 20(6), 337-344.

Hirata, T., Aiken, J., Hardman-Mountford, N., Smyth, T. J., \& Barlow, R. (2008). An absorption model to determine phytoplankton size classes from satellite ocean colour. Remote Sensing of Environment, 112, 3153-3159.

Hirata, T., Hardman-Mountford, N. J., Brewin, R. J. W., Aiken, J., Barlow, R., Suzuki, K., et al. (2011). Synoptic relationships between surface Chlorophyll- $a$ and diagnostic pigments specific to phytoplankton functional types. Biogeosciences, 8, 311-327. doi:10.5194/bg-8-311-2011.

Hooker, S. B., Van Heukelem, L., Thomas, C. S., Claustre, H., Ras, J., Barlow, R., et al. (2005). The second SeaWiFS HPLC analysis round robin experiment (SeaHARRE-2). NASA TM/2005-212785. Greenbelt, Maryland: NASA Goddard Space Flight Center.

International Ocean-Colour Coordinating Group (IOCCG) (1999). Status and plans fo satellite ocean-colour missions: Considerations for complementary missions. In J. Yoder (Ed.), Reports of the International Ocean-Color Coordinating Group, no. 2. Dartmouth, Nova Scotia.

International Ocean-Colour Coordinating Group (IOCCG) (2000). Remote sensing of ocean color in coastal, and optically-complex waters. In S. Sathyendranath (Ed.) Reports of the International Ocean-Color Coordinating Group, no. 3. Dartmouth, Nova Scotia.

Jeffrey, S. W., Mantoura, R. F. C., \& Wright, S. W. (1997). Phytoplankton pigments in oceanography. Paris: UNESCO Publishing.

Lewitus, A. J., White, D. L., Tymowski, R. G., Geesey, M. E., Hymel, S. N., \& Noble, P. A (2005). Adapting the CHEMTAX method for assessing phytoplankton taxonomic composition in southeastern U.S. estuaries. Estuaries, 28(1), 160-172.

Li, A., Stoecker, D. K., \& Adolf, J. E. (1999). Feeding, pigmentation, photosynthesis and growth of the mixotrophic dinoflagellate Gyrodinium galatheanum. Aquatic Microbial Ecology, 19(2), 163-176. doi:10.3354/ame019163.

Mackey, M. D., Mackey, D. J., Higgins, H. W., \& Wright, S. W. (1996). CHEMTAX-a program for estimating class abundances from chemical markers: Application to HPLC measurements of phytoplankton. Marine Ecology Progress Series, 144, 265-283.

Mannino, A., Russ, M. E., \& Hooker, S. B. (2008). Algorithm development and validation for satellite-derived distributions of DOC and CDOM in the U.S. Middle Atlantic Bight. Journal of Geophysical Research, 113, C07051. doi:10.1029/2007JC004493.

Marshall, H. G. (1984). Phytoplankton of the northeastern continental shelf of the United States in relation to abundance, composition, cell volume, seasonal, and regional assemblages. Rapports et Proces-Verbaux des Reunions, Conseil International pour l'Exploration de la Met, 183. (pp. 41-50).

Marshall, H. G. (1984). Phytoplankton distribution along the eastern coast of the USA Part V. Seasonal density and cell volume patterns for the northeastern continental shelf. Journal of Plankton Research, 6(1), 169-193.

Marshall, H. G., \& Alden, R. W. (1990). A comparison of phytoplankton assemblages and environmental relationships in three estuarine rivers of the lower Chesapeake Bay. Estuaries, 13, 287-300.

Marshall, H. G., \& Cohn, M. S. (1987). Phytoplankton distribution along the eastern coast of the USA. Part VI. Shelf waters between Cape Henry and Cape May. Journa of Plankton Research, 9(1), 139-149.
Marshall, H. G., Lacouture, R. V., Buchanan, C., \& Johnson, J. M. (2006). Phytoplankton assemblages associated with water quality and salinity regions in Chesapeake Bay, USA. Estuarine, Coastal and Shelf Science, 69, 10-18.

Marshall, H. G., \& Ranasinghe, A. (1989). Phytoplankton distribution along the eastern coast of the U.S.A. VII. Mean cell concentrations and standing crop. Continental Shelf Research, 9(2), 153-164.

McClain, C. R. (2009). A decade of satellite ocean color observations. Annual Review of Marine Science, 1, 19-42. doi:10.1146/annurev.marine.010908.163650.

Moisan, T. A., \& Mitchell, B. G. (1999). Photophysiological acclimation of Phaeocystis antarctica Karsten under light limitation. Limnology and Oceanography, 44(2), 247-258.

Moline, M. A., \& Blackwell, S. M. (2004). Episodic physical forcing and the structure of phytoplankton communities in the coastal waters of New Jersey. Journal of Geophysical Research, 109, C12S05. doi:10.1029/2003JC001985.

Montes-Hugo, M., Doney, S. C. Ducklow, H. W. Fraser, W., Martinson, D., Stammerjohn, S. E., et al. (2009). Recent changes in phytoplankton communities associated with rapid regional climate change along the western Antarctic Peninsula. Science, 323, 1470-1473.

O'Reilly, J. E., Maritorena, S., Mitchell, B. G., Siegel, D. A., Carder, K. L., Garver, S. A., et al. (1998). Ocean color algorithms for SeaWiFS. Journal of Geophysical Research, 103, $24937-24953$.

O'Reilly, J. E. \& Zetlin, C. (1998). Seasonal, horizontal, and vertical distribution of phytoplankton chlorophyll a in the northeast U.S. continental shelf ecosystem. NOAA Technical Report NMFS 139. Seattle, Washington: U.S. Department of Commerce.

O'Reilly, J. E., Maritorena, S., Siegel, D., O'Brien, M. C., Toole, D., Mitchell, B. G., et al. (2000). SeaWiFS postlaunch calibration and validation analyses, part 3. In S. B. Hooker, \& E. R. Firestone (Eds.), SeaWiFS post-launch technical report series. NASA technical memorandum 2000-206892, Volume 11, Greenbelt, Maryland: NASA Goddard Space Flight Center.

Pan, X., Mannino, A., Russ, M. E., \& Hooker, S. B. (2008). Remote sensing of the absorption coefficients and chlorophyll a concentration in the United States southern Middle Atlantic Bight from SeaWiFS and MODIS-Aqua. Journal of Geophysical Research, 113, C11022. doi:10.1029/2008JC004852.

Pan, X., Mannino, A., Russ, M. E., Hooker, S. B., \& Harding, L. W. (2010). Remote sensing of phytoplankton pigment distribution in the United States northeast coast. Remote Sensing of Environment, 114, 2403-2416. doi:10.1016/j.rse.2010.05.015.

Pan, X., \& Zimmerman, R. C. (2010). Modeling the vertical distributions of downwelling plane irradiance and diffuse attenuation coefficient in optically deep waters. Journal of Geophysical Research, 115, C08016. doi:10.1029/2009JC006039.

Reynolds, C. S., \& Walsby, A. E. (1975). Water blooms. Biological Reviews, 50, 437-481.

Richardson, A. J., \& Schoeman, D. S. (2004). Climate impact on plankton ecosystems in the northeast Atlantic. Science, 305, 1609-1612.

Roy, S., Platt, T., \& Sathyendranath, S. (2011). Modelling the time-evolution of phytoplankton size spectra from satellite remote sensing. ICES Journal of Marine Science, 68, 719-728. doi:10.1093/icesjms/fsq176.

Schlüter, L., Mohlenberg, F., Havskum, H., \& Larsen, S. (2000). The use of phytoplankton pigments for identifying and quantifying phytoplankton groups in coastal areas: Testing the influence of light and nutrients on pigment/chlorophyll a ratios. Marine Ecology Progress Series, 192, 49-63.

Smith, V. H. (2006). Responses of estuarine and coastal marine phytoplankton to nitrogen and phosphorus enrichment. Limnology and Oceanography, 51(1, part 2), 377-384

Townsend, D. W., \& Keller, M. D. (1996). Dimethylsulfide (DMS) and dimethylsulfoniopropionate (DMSP) in relation to phytoplankton in the Gulf of Maine. Marine Ecology Progress Series, 137, 229-241.

Uitz, J., Claustre, H., Griffiths, F. B., Ras, J., Garcia, N., \& Sandroni, V. (2009). A phytoplankton class-specific primary production model applied to the Kerguelen Islands region (Southern Ocean). Deep Sea Research I, 56(4), 541-560. doi:10.1016/j.dsr.2008.11.006.

Van Heukelem, L., \& Thomas, C. S. (2001). Computer-assisted high-performance liquid chromatography method development with applications to the isolation and analysis of phytoplankton pigments. Journal of Chromatography. A, 910, 31-49.

Vaulot, D., Eikrem, W., Viprey, M., \& Moreau, H. (2008). The diversity of small eukaryotic phytoplankton $(\leq 3 \mu \mathrm{m})$ in marine ecosystems. FNMS Microbiology Reviews, 32 (5), 795-820. doi:10.1111/j.1574-6976.2008.00121.x.

Venrick, E. L. (1978). How many cells to count. In A. Sournia (Ed.), Phytoplankton manual (pp. 167-180). Paris: UNESCO Publishing.

Vidussi, F., Claustre, H., Manca, B. B., Luchetta, A., \& Marty, J. C. (2001). Phytoplankton pigment distribution in relation to upper thermocline circulation in the eastern Mediterranean Sea during winter. Journal of Geophysical Research, 106(C9), 19939-19956.

Wright, S. W., Thomas, D. P., Marchant, H. J., Higgins, H. W., Mackey, M. D., \& Mackey, D. J. (1996). Analysis of phytoplankton of the Australian sector of the Southern Ocean: Comparisons of microscopy and size frequency data with interpretations of pigment HPLC data using the 'CHEMTAX' matrix factorization program. Marine Ecology Progress Series, 144, 285-298.

Zapata, M., Jeffrey, S. W., Wright, S. W., Rodríguez, F., Garrido, J. L., \& Clementson, L. (2004). Photosynthetic pigments in 37 species (65 strains) of Haptophyta: Implications for oceanography and chemotaxonomy. Marine Ecology Progress Series, $270,83-102$. 